\title{
New Analytical Study of the Effects Thermo-Diffusion, Diffusion-Thermo and Chemical Reaction of Viscous Fluid on Magneto Hydrodynamics Flow in Divergent and Convergent Channels
}

\author{
Abdul-Sattar J. A. Al-Saif', Abeer Majeed Jasim² \\ ${ }^{1}$ Department of Mathematics, College of Education for Pure Science, University of Basrah, Basrah, Iraq \\ ${ }^{2}$ Department of Mathematics, College of Science, University of Basrah, Basrah, Iraq \\ Email: sattaralsaif@yahoo.com, abeer.jassem@yahoo.com
}

How to cite this paper: Al-Saif, A.-S.J.A. and Jasim, A.M. (2019) New Analytical Study of the Effects Thermo-Diffusion, Diffusion-Thermo and Chemical Reaction of Viscous Fluid on Magneto Hydrodynamics Flow in Divergent and Convergent Channels. Applied Mathematics, 10, 268-300. https://doi.org/10.4236/am.2019.104020

Received: March 27, 2019

Accepted: April 26, 2019

Published: April 29, 2019

Copyright $\odot 2019$ by author(s) and Scientific Research Publishing Inc. This work is licensed under the Creative Commons Attribution International License (CC BY 4.0).

http://creativecommons.org/licenses/by/4.0/ c) (†) Open Access

\begin{abstract}
In this paper, the magneto hydrodynamic (MHD) flow of viscous fluid in a channel with non-parallel plates is studied. The governing partial differential equation was transformed into a system of dimensionless non-similar coupled ordinary differential equation. The transformed conservations equations were solved by using new algorithm. Basically, this new algorithm depends mainly on the Taylor expansion application with the coefficients of power series resulting from integrating the order differential equation. Results obtained from new algorithm are compared with the results of numerical Range-Kutta fourth-order algorithm with help of the shooting algorithm. The comparison revealed that the resulting solutions were excellent agreement. Thermo-diffusion and diffusion-thermo effects were investigated to analyze the behavior of temperature and concentration profile. Also the influences of the first order chemical reaction and the rate of mass and heat transfer were studied. The computed analytical solution result for the velocity, temperature and concentration distribution with the effect of various important dimensionless parameters was analyzed and discussed graphically.
\end{abstract}

\section{Keywords}

Thermo-Diffusion, Diffusion-Thermo, Chemical Reaction, Analytical

Approximate Solution, Mass and Heat Transfer, Magneto Hydrodynamics 


\section{Introduction}

The importance of thermal-diffusion and diffusion-thermo effects for various fluid flows has been studied by Eckert and Drake [1]. Olajuwon [2] examined convection heat and mass transfer in a hydromagnetic flow of a second grate fluid past a semi-infinite stretching sheet in the presence of thermal diffusion and thermal radiation. Kumar et al. [3] have investigated thermal diffusion and radiation effects on unsteady magneto hydrodynamics (MHD) flow through porous medium with variable temperature and mass diffusion in the presence of heat source or sink. Magnetohydrodynamics is the study of the interaction between magnetic fields and moving, conducting fluids [4] and the behavior of an electrically conducting fluid in the presence of a magnetic field. In this case, a force is produced inside the fluid which is proportional to fluid velocity and this force always opposes the flow. Another way to produce a force inside a flowing fluid, not known widely, is the application of an externally applied magnetic as well as an externally applied electric field. This force is called Lorentz force and can be generated by a strip wise arrangement of flush mounted electrodes and permanent magnets of alternating polarity and magnetization. The Lorentz force which acts parallel to the plate can either assist or oppose the flow. The idea of using a Lorentz force to stabilize a boundary layer flow over a flat plate belongs probably to Gailitis and Lielausis [5] [6]. It is a known fact that the temperature and concentration gradients present mass and energy fluxes, respectively. Concentration gradients result in Dufuor effect (diffusion-thermo) but Soret effect (thermal-diffusion) is due to temperature gradients. The heat and mass transfer with chemical reaction plays an important role in designing of chemical processing equipment, damage of crops due to frost, formulation and dispersion of fog. The mass transfer can be defined as a phenomenon when there is an escape of vapors into the atmosphere while heat transfer happens when there is heating or cooling of a liquid or fluid. That is, both of these phenomena play an important role in the industry. Because nonlinearity of the equations for these problem exact solutions is known, so many analytical techniques have been studied. Homotopy analysis method [7] [8] and Adomian's decomposition method [9] [10] [11] [12] are also analytical techniques used to solve the nonlinear equations. In this article the governing equations of the problem contain a system of partial differential equations which are transformed by usual transformation into a non-dimensional system of partial coupled non-linear differential equations. The purpose of this article is to investigate the diffusion-thermo and thermal diffusion effects on converging and diverging channel in the presence of chemical reaction. In present problem we apply a new technique to solve the equation governing the flow of viscous fluid in diverging and converging channels called a new algorithm. This new algorithm that includes the use of several steps, first integration and then we use Taylor expansion in addition to the last step involves extracting the value of derivatives. MHD, Soret, Dufour and chemical reaction effects are taken into account. Influences of physical parameters on 
temperature and concentration profiles are discussed for both diverging and converging channels with the help of graphs. The structure of this paper is organized as follows: Section 2 definitions of Mathematical formulation. Section 3 explains Description of the new scheme. Section 4, we apply new algorithm method to solve the magneto hydrodynamic (MHD) flow of viscous fluid in a channel with non-parallel plates show its ability and efficiency in finding new approximate solutions. Section 5 evidences that a new algorithm is converged through new theorems with its application. Section 6 discusses the effect of physical parameters on velocity, temperature, concentration profiles by help tables and graphics. Section 7 introduces conclusions of the present work.

\section{Mathematical Formulation}

Consider the flow of an incompressible fluid due to source or sink that is located at the intersection of two rigid plane walls angled $2 \alpha$ apart. Radial and symmetric nature of the flow is taken into consideration. Induced magnetic field is ignored and an applied magnetic field is considered that is applied across the flow direction. Under the aforesaid assumptions velocity field takes the form $V=\left[u_{r}, 0,0\right]$, where $u_{r}$ is a function of both $r$ and $\theta$. Soret and Dufour effects are also considered that are incorporated in energy and concentration equations respectively. The fluid is also assumed to be chemically reacting. Also, the temperature and concentration are also the function of both $r$ and $\theta$. While the results of angle opening $\alpha$ on concentration profile show that the increase in angle gives a decrease in concentration profile. The governing equations for mass, motion, energy, and mass transfer in polar coordinates under imposed assumptions become [13] [14] (Figure 1).

$$
\begin{gathered}
\frac{1}{r} \frac{\partial}{\partial r}\left(r u_{r}\right)=0, \\
u_{r} \frac{\partial u_{r}}{\partial r}=-\frac{1}{\rho} \frac{\partial p}{\partial r}+v\left[\frac{\partial^{2} u_{r}}{\partial r^{2}}+\frac{1}{r} \frac{\partial u_{r}}{\partial r}+\frac{1}{r^{2}} \frac{\partial^{2} u_{r}}{\partial \theta^{2}}-\frac{u_{r}}{r^{2}}\right]-\frac{\sigma B_{0}^{2}}{\rho} u_{r}, \\
-\frac{1}{\rho r} \frac{\partial p}{\partial \theta}+\frac{2 v}{r^{2}} \frac{\partial u_{r}}{\partial \theta}=0, \\
\rho c_{p} u_{r} \frac{\partial T}{\partial r}=k\left[\frac{\partial^{2} T}{\partial r^{2}}+\frac{1}{r} \frac{\partial T}{\partial r}+\frac{1}{r^{2}} \frac{\partial^{2} T}{\partial \theta^{2}}\right]+\mu\left[4\left(\frac{\partial u_{r}}{\partial r}\right)^{2}+\left(\frac{\partial u_{r}}{\partial \theta}\right)^{2}\right] \\
+\frac{D K_{T}}{C_{s}} \frac{\partial^{2} C}{\partial r^{2}}+\frac{1}{r} \frac{\partial C}{\partial r}+\frac{1}{r^{2}} \frac{\partial^{2} C}{\partial \theta^{2}} \\
u_{r} \frac{\partial C}{\partial r}=D\left[\frac{\partial^{2} C}{\partial r^{2}}+\frac{1}{r} \frac{\partial C}{\partial r}+\frac{1}{r^{2}} \frac{\partial^{2} C}{\partial \theta^{2}}\right]+\frac{D K_{T}}{T_{m}}\left[\frac{\partial^{2} T}{\partial r^{2}}+\frac{1}{r} \frac{\partial T}{\partial r}+\frac{1}{r^{2}} \frac{\partial^{2} T}{\partial \theta^{2}}\right]
\end{gathered}
$$

the boundary conditions are,

$$
u_{r}=U, \frac{\partial u_{r}}{\partial \theta}=0, \frac{\partial T}{\partial \theta}=0, \frac{\partial C}{\partial \theta}=0, \text { at } \theta=\alpha,
$$




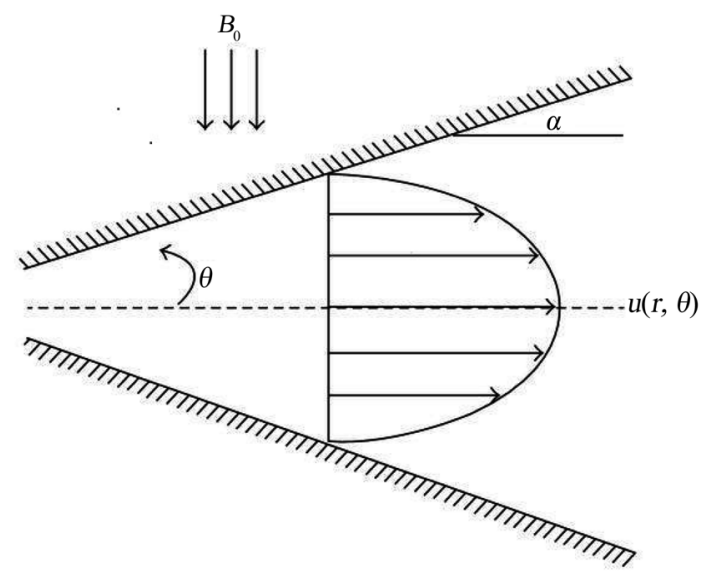

Figure 1. Schematic diagram of the flow problem.

$$
u_{r}=0, T=T_{m}, C=C_{w} \text {, at } \theta=\alpha,
$$

where $p$ is the fluid pressure, $v=\frac{\mu}{\rho}$ is kinematic viscosity, $\mathrm{D}$ in order are the specific heat and coefficient of mass diffusivity. $K, K_{T}, D$ correspondingly are the thermal conductivity, thermal-diffusion ratio and coefficient of mass diffusivity. Further $C_{s}, T_{w}, T_{m}, C_{w}, K_{1}$ represent the concentration susceptibility, temperature at wall, mean fluid temperature, concentration at the wall and the chemical reaction constant respectively. From the continuity Equation (1), we can write

$$
F(\theta)=r u_{r}(r, \theta)
$$

with the use of dimensionless parameters award [6]

$$
f(\eta)=\frac{F(\theta)}{r U}, \quad \eta=\frac{\theta}{\alpha}, \quad \beta(\eta)=\frac{T}{T_{w}}, \quad \phi(\eta)=\frac{C}{C_{w}} .
$$

Eliminating $p$ from Equations (1) and (2) using Equations (7) and (8), we get a system of nonlinear ordinary differential equation for the normalized velocity profile $f(\eta)$, temperature profile $\beta(\eta)$ and concentration profile $\phi(\eta)$

$$
\begin{gathered}
f^{\prime \prime \prime}(\eta)+2 \alpha \operatorname{Ref}(\eta) f^{\prime}(\eta)+(4-H a) \alpha^{2} f^{\prime}(\eta)=0 \\
\beta^{\prime \prime}(\eta)+E_{c} P_{r}\left[4 \alpha^{2} f^{2}(\eta)+\left(f^{\prime}(\eta)\right)^{2}\right]+D_{f} P_{r} \phi^{\prime \prime}(\eta)=0 \\
\phi^{\prime \prime}(\eta)+S_{c} S_{r} \beta^{\prime \prime}-S_{c} \gamma \alpha^{2} \phi(\eta)=0 \\
f(0)=1, f^{\prime}(0)=0, f(1)=0 \\
\beta^{\prime}(0)=0, \beta(1)=1 \\
\phi(0)=0, \phi(1)=1
\end{gathered}
$$

where,

$$
\operatorname{Re}=\frac{U r \alpha}{\mu}\left\{\begin{array}{l}
\text { divergent channel }: \alpha>0, U>0 \\
\text { convergent channel }: \alpha<0, U<0
\end{array}, H a=\sqrt{\frac{\sigma B_{0}^{2}}{\mu}}, P_{r}=\frac{\mu c_{p}}{K} .\right.
$$




$$
E_{c}=\frac{U^{2}}{c_{p} T_{w}}, \quad D_{f}=\frac{D K_{T} C_{w}}{v c_{p} C_{s} T_{w}}, S_{c}=\frac{v}{D}, \quad S_{r}=\frac{D K_{T} T_{w}}{v T_{w} C_{w}}, \text { represent Reynolds, }
$$

Hartmman, Prandtl, Eckert, Dufour, Schmidt and Soret number respectively while $\gamma=\frac{K_{1}}{v}$ is the first order chemical reaction parameter. The local Nusselt and Sherwood numbers are define by

$$
\begin{aligned}
& N u=\frac{\left.q_{w}\right|_{\eta=1}}{K T_{w}}=-\frac{1}{\alpha} \beta^{\prime}(1), \\
& S h=\frac{\left.M_{w}\right|_{\eta=1}}{D C_{w}}=-\frac{1}{\alpha} \phi^{\prime}(1) .
\end{aligned}
$$

\section{Description of the New Algorithm}

This section describes how to obtain a new scheme to calculate the coefficients of the power series solution resulting from solving nonlinear ordinary differential equations to find analytical-approximate solution. These coefficients are important basis to construct the solution formula, therefore they can be computed recursively by differentiation ways. To illustrative the computation and operations for these coefficients and derivation the new scheme, we summarized the detail a new outlook in the following steps.

Step (1): Consider the non-linear differential equation as follows:

$$
H\left(f(\eta), f^{\prime}(\eta), f^{\prime \prime}(\eta), f^{\prime \prime \prime}(\eta), \cdots, f^{(n-1)}(\eta), f^{(n)}(\eta)\right)=0,
$$

integrating Equation (13) with respect to $\eta$ on $[0, \eta]$ yield

$$
f(\eta)=f(0)+f^{\prime}(0) \eta+f^{\prime \prime}(0) \frac{\eta^{2}}{2 !}+\cdots+f^{(n-1)}(0) \frac{\eta^{n-1}}{(n-1) !}+L^{-1} G[f(\eta)],
$$

where,

$$
G[f(\eta)]=H\left(f(\eta), f^{\prime}(\eta), f^{\prime \prime}(\eta), \cdots, f^{(n-1)}(\eta)\right), L^{-1}=\int_{0}^{\eta} \int_{0}^{\eta} \cdots \int_{0}^{\eta}(\mathrm{d} \eta)^{n}
$$

Step (2): We take Taylor series expansion of the function $G[f(\eta)]$ about $\eta=\eta_{0}$ as follows

$$
G[f(\eta)]=\sum_{n=0}^{\infty} \frac{(\Delta \eta)^{n}}{n !} \frac{\mathrm{d}^{n} G\left(f_{0}(\eta)\right)}{\mathrm{d} \eta^{n}}
$$

rewriting the Equation (16)

$$
\begin{aligned}
G[f(\eta)]= & G\left[f_{0}(\eta)\right]+\frac{\Delta \eta}{1 !} G^{\prime}\left[f_{0}(\eta)\right]+\frac{(\Delta \eta)^{2}}{2 !} G^{\prime \prime}\left[f_{0}(\eta)\right] \\
& +\frac{(\Delta \eta)^{3}}{3 !} G^{\prime \prime \prime}\left[f_{0}(\eta)\right]+\cdots
\end{aligned}
$$

Now, we assume that $\Delta \eta=\max \left\{\eta, \eta_{0}\right\}$ and substituting Equation (17) in Equation (14), we obtain

$$
f(\eta)=f_{0}+f_{1}+f_{2}+f_{3}+f_{4}+\cdots,
$$


where,

$$
\begin{gathered}
f_{0}=f(0)+f^{\prime}(0) \eta+f^{\prime \prime}(0) \frac{\eta^{2}}{2 !}+\cdots+f^{(n-1)}(0) \frac{\eta^{(n-1)}}{(n-1) !}, \quad f_{1}=L^{-1} G\left[f_{0}(\eta)\right] \\
f_{2}=L^{-1} \frac{\max \left\{\eta, \eta_{0}\right\}}{1 !} G^{\prime}\left[f_{0}(\eta)\right], \quad f_{3}=L^{-1} \frac{\left(\max \left\{\eta, \eta_{0}\right\}\right)^{2}}{2 !} G^{\prime \prime}\left[f_{0}(\eta)\right] \\
f_{4}=L^{-1} \frac{\left(\max \left\{\eta, \eta_{0}\right\}\right)^{3}}{3 !} G^{\prime \prime \prime}\left[f_{0}(\eta)\right] \cdots
\end{gathered}
$$

Step (3): We focus on computing the derivatives of $G$ with respect to $\eta$ which is the crucial part of the proposed method. Let start calculating

$$
\begin{aligned}
& G[f(\eta)], G^{\prime}[f(\eta)], G^{\prime \prime}[f(\eta)], G^{\prime \prime \prime}[f(\eta)], \cdots . \\
& G[f(\eta)]=H\left(f(\eta), f^{\prime}(\eta), f^{\prime \prime}(\eta), f^{\prime \prime \prime}(\eta), f^{\prime \prime \prime \prime}(\eta), \cdots, f^{(n-1)}(\eta)\right), \\
& G^{\prime}[f(\eta)]=\frac{\mathrm{d} G[f(\eta)]}{\mathrm{d} \eta}=G_{1 f} \cdot f_{\eta}+G_{1 f^{\prime}} \cdot\left(f_{\eta}\right)^{\prime}+\cdots+G_{f^{(n-1)}} \cdot\left(f_{\eta}\right)^{(n-1)} \\
& G^{\prime \prime}[f(\eta)]=\frac{\mathrm{d}^{2} G[f(\eta)]}{\mathrm{d} z^{2}} \\
& =G_{f f} \cdot\left(f_{\eta}\right)^{2}+G_{f f^{\prime}} \cdot\left(f_{\eta}\right)^{\prime} f_{\eta}+G_{f f^{\prime \prime}} \cdot f_{\eta}\left(f_{\eta}\right)^{\prime \prime}+\cdots \\
& +G_{f f^{(n-1)}} \cdot\left(f_{\eta}\right)\left(f_{\eta}\right)^{(n-1)}+G_{f} \cdot f_{\eta \eta}+G_{f f^{\prime}} \cdot\left(f_{\eta}\right)^{\prime} \cdot f_{\eta}+G_{f f^{\prime}} \cdot\left(f_{\eta}\right)^{\prime 2} \\
& +\cdots+G_{f f^{(n-1)}} \cdot\left(f_{\eta}\right)^{\prime}\left(f_{\eta}\right)^{(n-1)}+G_{f^{\prime}} \cdot\left(f_{\eta \eta}\right)^{\prime}+G_{f^{\prime \prime}} \cdot\left(f_{\eta}\right)^{\prime \prime} \cdot f_{\eta} \\
& +G_{f^{\prime \prime} y^{\prime}} \cdot\left(f_{\eta}\right)^{\prime}\left(f_{\eta}\right)^{\prime \prime}+G_{f^{\prime y^{\prime \prime}}} \cdot\left(f_{\eta}\right)^{\prime \prime 2}+G_{f^{\prime y^{\prime}(n-1)}} \cdot\left(f_{\eta}\right)^{\prime \prime}\left(f_{\eta}\right)^{(n-1)} \\
& +G f^{\prime \prime} \cdot\left(f_{\eta \eta}\right)^{\prime \prime}+\cdots+G_{f^{(n-1)} f} \cdot\left(f_{\eta}\right)^{(n-1)} \cdot f_{\eta}+G_{f^{(n-1)} f^{\prime}} \cdot\left(f_{\eta}\right)^{(n-1)} \cdot\left(f_{\eta}\right)^{\prime} \\
& +\cdots+G_{f^{(n-1)} f^{(n-1)}} \cdot\left(f_{\eta}\right)^{(n-1) 2}+G_{f^{(n-1)}} \cdot\left(f_{\eta \eta}\right)^{(n-1)} \\
& G^{\prime \prime \prime}[f(\eta)]=\frac{\mathrm{d}^{3} G[f(\eta)]}{\mathrm{d} \eta^{3}} \\
& =G_{f f f} \cdot\left(f_{\eta}\right)^{3}+G_{f f f^{\prime}} \cdot\left(f_{\eta}\right)^{2}\left(f_{\eta}\right)^{\prime}+\cdots+G_{f f f^{(n-1)}} \cdot\left(f_{\eta}\right)^{2} \cdot\left(f_{\eta}\right)^{(n-1)} \\
& +G_{f f} \cdot 2\left(f_{\eta}\right) \cdot f_{\eta \eta}+G_{f f f^{\prime}} \cdot\left(f_{\eta}\right)^{\prime}\left(f_{\eta}\right)^{2}+G_{f f f^{\prime}} \cdot\left(f_{\eta}\right)^{\prime 2}\left(f_{\eta}\right)+\cdots \\
& +G_{f f f^{(n-1)}} \cdot\left(f_{\eta}\right)^{\prime}\left(f_{\eta}\right) \cdot\left(f_{\eta}\right)^{(n-1)}+G_{f f^{\prime}} \cdot\left[\left(f_{\eta \eta}\right)^{\prime} \cdot f_{\eta}+\left(f_{\eta}\right)^{\prime} \cdot f_{\eta \eta}\right] \\
& +G_{f f^{\prime \prime} \eta^{\prime}} \cdot\left(f_{\eta}\right)^{\prime \prime}\left(f_{\eta}\right)^{2}+G_{f f^{\prime \prime \prime} f^{\prime}} \cdot\left(f_{\eta}\right)^{\prime \prime}\left(f_{\eta}\right) \cdot\left(f_{\eta}\right)^{\prime}+\cdots \\
& +G_{f f f^{\prime}(n-1)} \cdot\left(f_{\eta}\right)^{\prime \prime}\left(f_{\eta}\right) \cdot\left(f_{\eta}\right)^{(n-1)}+G_{f f^{\prime \prime}} \cdot\left[f_{\eta \eta} \cdot\left(f_{\eta}\right)^{\prime \prime}+f_{\eta} \cdot\left(f_{\eta \eta}\right)^{\prime \prime}\right]+\cdots \\
& +G_{f f^{(n-1)} f} \cdot\left(f_{\eta}\right)^{2} \cdot\left(f_{\eta}\right)^{(n-1)}+G_{f f^{(n-1)} f^{\prime}} \cdot\left(f_{\eta}\right) \cdot\left(f_{\eta}\right)^{\prime} \cdot\left(f_{\eta}\right)^{(n-1)}+\cdots \\
& +G_{f f(n-1) f^{(n-1)}} \cdot\left(f_{\eta}\right) \cdot\left(f_{\eta}\right)^{(n-1) 2}+G_{f f^{(n-1)}} \cdot\left[\left(f_{\eta \eta}\right) \cdot\left(f_{\eta}\right)^{(n-1)}+\left(f_{\eta}\right)\left(f_{\eta \eta}\right)^{(n-1)}\right]
\end{aligned}
$$




$$
\begin{aligned}
& +G_{f f} \cdot f_{\eta \eta} \cdot\left(f_{\eta}\right)+G_{f f^{\prime}} \cdot f_{\eta \eta} \cdot\left(f_{\eta}\right)^{\prime}+\cdots+G_{f f^{(n-1)}} \cdot f_{\eta \eta} \cdot\left(f_{\eta}\right)^{(n-1)}+G_{\eta} \cdot f_{\eta \eta \eta} \\
& +G_{f f f f} \cdot\left(f_{\eta}\right)^{\prime}\left(f_{\eta}\right)^{2}+G_{f f f} \cdot\left(f_{\eta}\right)^{\prime 2}\left(f_{\eta}\right)+\cdots+G_{f f f^{(n-1)}} \cdot\left(f_{\eta}\right)^{\prime}\left(f_{\eta}\right) \cdot\left(f_{\eta}\right)^{(n-1)} \\
& +G_{f f} \cdot\left[\left(f_{\eta \eta}\right)^{\prime} \cdot f_{\eta}+\left(f_{\eta}\right)^{\prime} \cdot f_{\eta \eta}\right]+G_{f f y} \cdot\left(f_{\eta}\right)^{\prime 2} \cdot f_{z}+G_{f f f^{\prime}} \cdot\left(f_{\eta}\right)^{\prime 3}+\cdots \\
& +G_{f f f f^{(n-1)}} \cdot\left(f_{\eta}\right)^{\prime 2} \cdot\left(f_{\eta}\right)^{(n-1)}+G_{f f^{\prime}} \cdot 2\left(f_{\eta}\right)^{\prime} \cdot\left(f_{\eta \eta}\right)^{\prime}+\cdots \\
& +G_{f^{(n-1)} f^{(n-1)} f} \cdot\left(f_{\eta}\right)^{(n-1) 2} \cdot f_{\eta}+G_{f^{(n-1)} f^{(n-1)} f^{\prime}} \cdot\left(f_{\eta}\right)^{(n-1) 2} \cdot\left(f_{\eta}\right)^{\prime}+\cdots \\
& \quad+G_{f^{(n-1)} f^{(n-1)} f^{(n-1)}} \cdot\left(f_{\eta}\right)^{(n-1) 3}+G_{f^{(n-1)} f^{(n-1)}} \cdot 2 \cdot\left(f_{\eta}\right)^{(n-1)} \cdot\left(f_{\eta \eta}\right)^{(n-1)} \\
& \quad+G_{f^{(n-1)} f} \cdot\left(f_{\eta \eta}\right)^{(n-1)} \cdot f_{\eta}+G_{f^{(n-1)} f^{\prime}} \cdot\left(f_{\eta \eta}\right)^{(n-1)} \cdot\left(f_{\eta}\right)^{\prime}+\cdots \\
& +G_{f^{(n-1)} f^{(n-1)}} \cdot\left(f_{\eta \eta}\right)^{(n-1)} \cdot\left(f_{\eta}\right)^{(n-1)}+G_{f^{(n-1)}} \cdot\left(f_{\eta \eta \eta}\right)^{(n-1)}
\end{aligned}
$$

The calculations are more complicated in the second and third derivatives because of the product rules. Consequently, the systematic structure on calculation is extremely important. Fortunately, due to the assumption that the operator $G$ and the solution $f$ are analytic functions, then the mixed derivatives are equivalence.

We note that the derivatives function to $f$ unknown, so we suggest the following hypothesis

$$
\begin{gathered}
f_{\eta}=f_{1}=L^{-1} G\left[f_{0}(\eta)\right], \quad f_{\eta \eta}=f_{2}=L^{-1} \frac{\max \left\{\eta, \eta_{0}\right\}}{1 !} G^{\prime}\left[f_{0}(\eta)\right] \\
f_{\eta \eta \eta}=f_{3}=L^{-1} \frac{\left(\max \left\{\eta, \eta_{0}\right\}\right)^{2}}{2 !} G^{\prime \prime}\left[f_{0}(\eta)\right] \\
f_{\eta \eta \eta \eta}=f_{4}=L^{-1} \frac{\left(\max \left\{\eta, \eta_{0}\right\}\right)^{3}}{3 !} G^{\prime \prime \prime}\left[f_{0}(\eta)\right] \\
f_{\eta \eta \eta \eta \eta}=f_{5}=L^{-1} \frac{\left(\max \left\{\eta, \eta_{0}\right\}\right)^{4}}{4 !} G^{\prime \prime \prime \prime}\left[f_{0}(\eta)\right], \cdots
\end{gathered}
$$

Therefore Equations (20)-(23) are evaluated by

$$
\begin{gathered}
G\left[f_{0}(\eta)\right]=H\left(f_{0}(\eta), f_{0}^{\prime}(\eta), f_{0}^{\prime \prime}(\eta), \cdots, f_{0}^{(n-1)}(\eta)\right), \\
G^{\prime}\left[f_{0}(\eta)\right]=G_{f_{0}} \cdot f_{1}+G_{f_{0}^{\prime}} \cdot\left(f_{1}\right)^{\prime}+G_{f_{0}^{\prime \prime}} \cdot\left(f_{1}\right)^{\prime \prime}+\cdots+G_{f_{0}^{(n-1)}} \cdot\left(f_{1}\right)^{(n-1)}, \\
G^{\prime \prime}\left[f_{0}(\eta)\right] \\
=G_{f_{0} f_{0}} \cdot\left(f_{1}\right)^{2}+G_{f_{0} f_{0}^{\prime}} \cdot\left(f_{1}\right)^{\prime} f_{1}+G_{f_{0} f_{0}^{\prime \prime}} \cdot f_{1}\left(f_{1}\right)^{\prime \prime}+\cdots+G_{f_{0} f_{0}^{(n-1)}} \cdot\left(f_{1}\right)\left(f_{1}\right)^{(n-1)} \\
+G_{f_{0}} \cdot f_{2}+G_{f_{0}^{\prime} f_{0}} \cdot\left(f_{1}\right)^{\prime} \cdot f_{1}+G_{f_{0}^{\prime} f_{0}^{\prime}} \cdot\left(f_{1}\right)^{\prime 2}+\cdots+G_{f_{0}^{\prime} f_{0}^{(n-1)}} \cdot\left(f_{1}\right)^{\prime}\left(f_{1}\right)^{(n-1)} \\
+G_{f_{0}^{\prime}} \cdot\left(f_{2}\right)^{\prime}+G_{f_{0}^{\prime \prime} f_{0}} \cdot\left(f_{1}\right)^{\prime \prime} \cdot f_{1}+G_{f_{0}^{\prime \prime} f_{0}} \cdot\left(f_{1}\right)^{\prime}\left(f_{1}\right)^{\prime \prime}+G_{f_{0}^{\prime \prime} f_{0}^{\prime \prime}} \cdot\left(f_{1}\right)^{\prime \prime 2}
\end{gathered}
$$




$$
\begin{aligned}
& +\cdots+G_{f_{0}^{\prime \prime} f_{0}^{(n-1)}} \cdot\left(f_{1}\right)^{\prime \prime}\left(f_{1}\right)^{(n-1)}+G_{f_{0}^{\prime \prime}} \cdot\left(f_{2}\right)^{\prime \prime}+G_{f^{(n-1)} f} \cdot\left(f_{1}\right)^{(n-1)} \cdot f_{1} \\
& +G_{f_{0}^{(n-1)} f_{0}^{\prime}} \cdot\left(f_{1}\right)^{(n-1)} \cdot\left(f_{1}\right)^{\prime}+\cdots+G_{f_{0}^{(n-1)} f_{0}^{(n-1)}} \cdot\left(f_{1}\right)^{(n-1) 2}+\cdots+G_{f_{0}^{(n-1)}} \cdot\left(f_{2}\right)^{(n-1)} \text {, } \\
& G^{\prime \prime \prime}\left[f_{0}(\eta)\right] \\
& =G_{f_{0} f_{0} f_{0}} \cdot\left(f_{1}\right)^{3}+G_{f_{0} f_{0} f_{0}^{\prime}} \cdot\left(f_{1}\right)^{2}\left(f_{1}\right)^{\prime}+\cdots+G_{f_{0} f_{0} f_{0}^{(n-1)}} \cdot\left(f_{1}\right)^{2} \cdot\left(f_{1}\right)^{(n-1)} \\
& +G_{f_{0} f_{0}} \cdot 2\left(f_{1}\right) \cdot f_{2}+G_{f_{0} f_{0}^{\prime} f_{0}} \cdot\left(f_{1}\right)^{\prime}\left(f_{1}\right)^{2}+G_{f_{0} f_{0}^{\prime} f_{0}^{\prime}} \cdot\left(f_{1}\right)^{\prime 2}\left(f_{1}\right)+\cdots \\
& +G_{f_{0} f_{0}^{\prime} f_{0}^{(n-1)}} \cdot\left(f_{1}\right)^{\prime}\left(f_{1}\right) \cdot\left(f_{1}\right)^{(n-1)}+G_{f_{0} f_{0}^{\prime}} \cdot\left[\left(f_{2}\right)^{\prime} \cdot f_{z}+\left(f_{1}\right)^{\prime} \cdot f_{2}\right] \\
& +G_{f_{0} f_{0}^{\prime \prime} f_{0}} \cdot\left(f_{1}\right)^{\prime \prime}\left(f_{1}\right)^{2}+G_{f_{0} f_{0}^{\prime \prime} f_{0}} \cdot\left(f_{1}\right)^{\prime \prime}\left(f_{1}\right) \cdot\left(f_{1}\right)^{\prime}+\cdots \\
& +G_{f_{0} f_{0}^{\prime \prime} f_{0}^{(n-1)}} \cdot\left(f_{1}\right)^{\prime \prime}\left(f_{1}\right) \cdot\left(f_{1}\right)^{(n-1)}+G_{f_{0} f_{0}^{\prime \prime}} \cdot\left[f_{2} \cdot\left(f_{1}\right)^{\prime \prime}+f_{1} \cdot\left(f_{2}\right)^{\prime \prime}\right]+\cdots \\
& +G_{f_{0} f_{0}^{(n-1)} f_{0}} \cdot\left(f_{1}\right)^{2} \cdot\left(f_{1}\right)^{(n-1)}+G_{f_{0} f_{0}^{(n-1)} f_{0}^{\prime}} \cdot\left(f_{1}\right) \cdot\left(f_{1}\right)^{\prime} \cdot\left(f_{1}\right)^{(n-1)}+\cdots \\
& +G_{f_{0} f_{0}^{(n-1)} f_{0}^{(n-1)}} \cdot\left(f_{1}\right)+\cdots+G_{f_{0} f_{0}^{(n-1)}} \cdot f_{2} \cdot\left(f_{1}\right)^{(n-1)}+G_{f_{0}} \cdot f_{3}+G_{f_{0}^{\prime} f_{0} f_{0}} \cdot\left(f_{1}\right)^{\prime}\left(f_{1}\right)^{2} \\
& +G_{f_{0}^{\prime} f_{0}^{\prime} f_{0}} \cdot\left(f_{1}\right)^{\prime 2}\left(f_{1}\right)+\cdots+G_{f_{0}^{\prime} f_{0} f_{0}^{(n-1)}} \cdot\left(f_{1}\right)^{\prime}\left(f_{1}\right) \cdot\left(f_{1}\right)^{(n-1)} \\
& +G_{f_{0}^{\prime} f_{0}} \cdot\left[\left(f_{2}\right)^{\prime} \cdot f_{1}+\left(f_{1}\right)^{\prime} \cdot f_{1}\right]+G_{f_{0}^{\prime} f_{0}^{\prime} f_{0}} \cdot\left(f_{1}\right)^{\prime 2} \cdot f_{1}+G_{f_{0}^{\prime} f_{0}^{\prime} f_{0}^{\prime}} \cdot\left(f_{1}\right)^{\prime 3}+\cdots \\
& +G_{f_{0}^{\prime} f_{0}^{\prime} f_{0}^{(n-1)}} \cdot\left(f_{1}\right)^{\prime 2} \cdot\left(f_{1}\right)^{(n-1)}+G_{f_{0}^{\prime} f_{0}^{\prime}} \cdot 2\left(f_{1}\right)^{\prime}\left(f_{2}\right)^{\prime}+\cdots+G_{f_{0}^{(n-1)} f_{0}^{(n-1)} f_{0}} \cdot\left(f_{1}\right)^{\prime 3} \\
& +\cdots+G_{f_{0}^{\prime} f_{0}^{\prime} f_{0}^{(n-1)}} \cdot\left(f_{1}\right)^{\prime 2} \cdot\left(f_{1}\right)^{(n-1)}+G_{f_{0}^{\prime} f_{0}^{\prime}} \cdot 2\left(f_{1}\right)^{\prime} \cdot\left(f_{2}\right)^{\prime}+\cdots \\
& +G_{f_{0}^{(n-1)} f_{0}^{(n-1)} f_{0}} \cdot\left(f_{1}\right)^{(n-1) 2} \cdot f_{1}+G_{f_{0}^{(n-1)} f_{0}^{(n-1)} f_{0}^{\prime}} \cdot\left(f_{1}\right)^{(n-1) 2} \cdot\left(f_{1}\right)^{\prime}+\cdots \\
& +G_{f_{0}^{(n-1)} f_{0}^{(n-1)} f_{0}^{(n-1)}} \cdot\left(f_{1}\right)^{(n-1) 3}+G_{f_{0}^{(n-1)} f_{0}^{(n-1)}} \cdot 2 \cdot\left(f_{1}\right)^{(n-1)} \cdot\left(f_{2}\right)^{(n-1)} \\
& +G_{f_{0}^{(n-1)} f_{0}} \cdot\left(f_{2}\right)^{(n-1)} \cdot f_{1}+G_{f_{0}^{(n-1)} f_{0}^{\prime}} \cdot\left(f_{2}\right)^{(n-1)} \cdot\left(f_{1}\right)^{\prime}+\cdots \\
& +G_{f_{0}^{(n-1)} f_{0}^{(n-1)}} \cdot\left(f_{2}\right)^{(n-1)} \cdot\left(f_{1}\right)^{(n-1)}+G_{f_{0}^{(n-1)}} \cdot\left(f_{3}\right)^{(n-1)}
\end{aligned}
$$

Step (4): Substituting Equations (25)-(28) in Equation (18) we get the required analytical-approximate solution for the Equation (13).

\section{Application of the New Algorithm to the Magneto Hydrodynamic (MHD) Flow of Viscous Fluid in a Channel with Non-Parallel Plates}

The new algorithm described in the previous section can be used as a powerful solver to the nonlinear differential Equations (9)-(10) and to find new an analytical-approximate solution. From step (1) we have

$$
f(\eta)=f(0)+f^{\prime}(0) \eta+f^{\prime \prime}(0) \frac{\eta^{2}}{2 !}+L_{1}^{-1}\left[-2 \alpha \operatorname{Ref}(\eta) f^{\prime}(\eta)-(4-H a) \alpha^{2} f^{\prime}(\eta)\right],
$$




$$
\begin{gathered}
\beta(\eta)=\beta(0)+\beta^{\prime}(0) \eta+L_{2}^{-1}\left[-E_{c} P_{r}\left[4 \alpha^{2} f^{2}(\eta)-\left(f^{\prime}(\eta)\right)^{2}\right]-D_{f} P_{r} \phi^{\prime \prime}(\eta)\right], \\
\phi(\eta)=\phi(0)+\phi^{\prime}(0) \eta+L_{3}^{-1}\left[-S_{c} S_{r} \beta^{\prime \prime}+S_{c} \gamma \alpha^{2} \phi(\eta)\right],
\end{gathered}
$$

rewrite the Equation (29) as follows

$$
\begin{gathered}
f(\eta)=A_{1}+A_{2} \eta+A_{3} \frac{\eta^{2}}{2 !}+L^{-1} G_{1}[f(\eta)], \\
\beta(\eta)=B_{1}+B_{2} \eta+L^{-1} G_{2}[\beta(\eta)], \\
\phi(\eta)=C_{1}+C_{2} \eta+L^{-1} G_{3}[\phi(\eta)],
\end{gathered}
$$

where,

$$
\begin{gathered}
A_{1}=f(0), \quad A_{2}=f^{\prime}(0), \quad A_{3}=f^{\prime \prime}(0), \\
B_{1}=\beta(0), \quad B_{2}=\beta^{\prime}(0), \quad C_{1}=\phi(0), \quad C_{2}=\phi^{\prime}(0), \\
G_{1}[f]=-2 \alpha \operatorname{Ref}(\eta) f^{\prime}(\eta)-(4-H a) \alpha^{2} f^{\prime}(\eta), \\
G_{2}[\beta]=-E_{c} P_{r}\left[4 \alpha^{2} f^{2}(\eta)-\left(f^{\prime}(\eta)\right)^{2}\right]-D_{f} P_{r} \phi^{\prime \prime}(\eta), \\
G_{3}[\phi]=-S_{c} S_{r} \beta^{\prime \prime}+S_{c} \gamma \alpha^{2} \phi(\eta), \\
\text { and } L_{1}^{-1}(.)=\int_{0}^{\eta} \int_{0}^{\eta} \int_{0}^{\eta}(\mathrm{d} \eta)^{3}, L_{2}^{-1}(.)=L_{3}^{-1}(.)=\int_{0}^{\eta} \int_{0}^{\eta}(\mathrm{d} \eta)^{2} .
\end{gathered}
$$

From the boundary conditions the Equation (30) becomes

$$
\begin{gathered}
f(\eta)=1+A_{3} \frac{\eta^{2}}{2 !}+L^{-1} G_{1}[f(\eta)], \\
\beta(\eta)=B_{1}+L^{-1} G_{2}[\beta(\eta)], \\
\phi(\eta)=C_{1}+L^{-1} G_{3}[\phi(\eta)] .
\end{gathered}
$$

From step (2) suppose that $\Delta \eta=\max \{1,0\}=1$, yield

$$
\begin{gathered}
f_{0}=1+A_{3} \frac{\eta^{2}}{2 !} \quad f_{1}=L_{1}^{-1} G_{1}\left[f_{0}(\eta)\right], \quad f_{2}=L_{2}^{-1} G_{1}^{\prime}\left[f_{0}(\eta)\right], \cdots, \\
\beta_{0}=B_{1}, \quad \beta_{1}=L_{2}^{-1} G_{2}\left[\beta_{0}(\eta)\right], \quad \beta_{2}=L_{2}^{-1} G_{2}^{\prime}\left[\beta_{0}(\eta)\right], \cdots, \\
f_{0}=C_{1}, \quad \phi_{1}=L_{3}^{-1} G_{3}\left[\phi_{0}(\eta)\right], \quad \phi_{2}=L_{3}^{-1} G_{3}^{\prime}\left[\phi_{0}(\eta)\right], \cdots,
\end{gathered}
$$

and the analytical-approximate solution are

$$
\begin{gathered}
f(\eta)=f_{0}+f_{1}+f_{2}+f_{3}+\cdots \\
\beta(\eta)=\beta_{0}+\beta_{1}+\beta_{2}+\beta_{3}+\cdots \\
\phi(\eta)=\phi_{0}+\phi_{1}+\phi_{2}+\phi_{3}+, \cdots
\end{gathered}
$$

From step (3) yields

$$
\begin{gathered}
G_{1}[f(\eta)]=-2 \alpha \operatorname{Ref}(\eta) f^{\prime}(\eta)-(4-H a) \alpha^{2} f^{\prime}(\eta), \\
G_{2}[\beta(\eta)]=-E_{c} P_{r}\left[4 \alpha^{2} f^{2}(\eta)-\left(f^{\prime}(\eta)\right)^{2}\right]-D_{f} P_{r} \phi^{\prime \prime}(\eta),
\end{gathered}
$$




$$
\begin{aligned}
& G_{3}[\phi(\eta)]=-S_{c} S_{r} \beta^{\prime \prime}+S_{c} \gamma \alpha^{2} \phi(\eta), \\
& G_{1}^{\prime}[f(\eta)]=\frac{\mathrm{d} G_{1}[f(\eta)]}{\mathrm{d} \eta}=G_{1 f} \cdot f_{\eta}+G_{1 f^{\prime}} \cdot\left(f_{\eta}\right)^{\prime}, \\
& G_{2}^{\prime}[\beta(\eta)]=\frac{\mathrm{d} G_{2}[\beta(\eta)]}{\mathrm{d} \eta}=G_{2 f} \cdot f_{\eta}+G_{2 f^{\prime}} \cdot\left(f_{\eta}\right)^{\prime}+G_{2} \phi^{\prime \prime} \cdot\left(\phi_{\eta}\right)^{\prime \prime}, \\
& G_{3}^{\prime}[\phi(\eta)]=\frac{\mathrm{d} G_{3}[\phi(\eta)]}{\mathrm{d} \eta}=G_{3 \beta^{\prime \prime}} \cdot\left(\beta_{\eta}\right)^{\prime \prime}+G_{3 \phi} \cdot\left(\phi_{\eta}\right), \\
& G_{1}^{\prime \prime}[f(\eta)]=\frac{\mathrm{d}^{2} G_{1}[f(\eta)]}{\mathrm{d} \eta^{2}} \\
& =G_{f f} \cdot\left(f_{\eta}\right)^{2}+2 \cdot G_{f f^{\prime}} \cdot f_{\eta}\left(f_{\eta}\right)^{\prime}+G_{f f^{\prime}} \cdot\left(f_{\eta}\right)^{\prime 2}+G_{f} \cdot f_{\eta \eta}+G_{f^{\prime}} \cdot\left(f_{\eta \eta}\right)^{\prime} \\
& G_{2}^{\prime \prime}[\beta(\eta)]=\frac{\mathrm{d}^{2} G_{2}[\beta(\eta)]}{\mathrm{d} \eta^{2}} \\
& =G_{f f} \cdot\left(f_{\eta}\right)^{2}+2 \cdot G_{f f^{\prime}} \cdot f_{\eta}\left(f_{\eta}\right)^{\prime}+G_{f f^{\prime}} \cdot\left(f_{\eta}\right)^{\prime 2}+G_{f} \cdot f_{\eta \eta}+G_{f^{\prime}} \cdot\left(f_{\eta \eta}\right)^{\prime} \\
& +G_{2 \phi^{\prime \prime} \phi^{\prime \prime}} \cdot\left(\phi_{\eta}\right)^{\prime \prime 2}+2 \cdot G_{2 \phi^{\prime \prime} f} \cdot\left(\phi_{\eta}\right)^{\prime \prime} \cdot f_{\eta}+2 \cdot G_{2 \phi^{\prime \prime} f^{\prime}} \cdot\left(\phi_{\eta}\right)^{\prime \prime} \cdot f_{\eta}^{\prime}+G_{2 \phi^{\prime \prime}} \cdot\left(\phi_{\eta \eta}\right)^{\prime \prime}, \\
& G_{3}^{\prime \prime}[\phi(\eta)]=\frac{\mathrm{d}^{2} G_{3}[\phi(\eta)]}{\mathrm{d} \eta^{2}} \\
& =G_{3 \beta^{\prime \prime} \beta^{\prime \prime}} \cdot\left(\beta_{\eta}\right)^{\prime \prime 2}+G_{3 \phi \phi} \cdot\left(\phi_{\eta}\right)^{2}+2 G_{3 \beta^{\prime \prime} \phi} \cdot\left(\beta_{\eta}\right)^{\prime \prime} \cdot \phi_{\eta}+G_{3 \phi} \cdot\left(\phi_{\eta \eta}\right)+G_{3 \beta^{\prime \prime}} \cdot\left(\beta_{\eta \eta}\right)^{\prime \prime} \text {, } \\
& G_{1}^{\prime \prime \prime}[f(\eta)]=\frac{\mathrm{d}^{3} G_{1}[f(\eta)]}{\mathrm{d} \eta^{3}} \\
& =G_{1 f f f}\left(f_{\eta}\right)^{3}+3 \cdot G_{1 f f f^{\prime}}\left(f_{\eta}\right)^{2} \cdot\left(f_{\eta}\right)^{\prime}+3 \cdot G_{1 f f f^{\prime}} \cdot\left(f_{\eta}\right)\left(f_{\eta}\right)^{\prime 2} \\
& +G_{1 f f f^{\prime}} \cdot\left(f_{\eta}\right)^{\prime 3}+3 \cdot G_{1 f f} \cdot f_{\eta \eta} \cdot f_{\eta}+3 \cdot G_{1 f f^{\prime}} \cdot f_{\eta \eta} \cdot\left(f_{\eta}\right)^{\prime}+G_{1 f} \cdot f_{\eta \eta \eta} \\
& +3 \cdot G_{1 f^{\prime}} \cdot\left(f_{\eta \eta}\right)^{\prime}\left(f_{\eta}\right)+3 \cdot G_{1 f f^{\prime}} \cdot\left(f_{\eta \eta}\right)^{\prime}\left(f_{\eta}\right)^{\prime}+G_{1 f^{\prime}} \cdot\left(f_{\eta \eta \eta}\right)^{\prime}, \\
& G_{2}^{\prime \prime \prime}[\beta(\eta)]=\frac{\mathrm{d}^{3} G_{1}[\beta(\eta)]}{\mathrm{d} \eta^{3}} \\
& =G_{2 f f f^{\prime}}\left(f_{\eta}\right)^{3}+3 \cdot G_{2 f f f^{\prime}}\left(f_{\eta}\right)^{2} \cdot\left(f_{\eta}\right)^{\prime}+3 \cdot G_{2 f f f^{\prime}} \cdot\left(f_{\eta}\right)\left(f_{\eta}\right)^{\prime 2}+G_{2 f f f^{\prime}} \cdot\left(f_{\eta}\right)^{\prime 3} \\
& +3 \cdot G_{2 f f} \cdot f_{\eta \eta} \cdot f_{\eta}+3 \cdot G_{2 f f^{\prime}} \cdot f_{\eta \eta} \cdot\left(f_{\eta}\right)^{\prime}+G_{2 f} \cdot f_{\eta \eta \eta}+3 \cdot G_{2 f y} \cdot\left(f_{\eta \eta}\right)^{\prime}\left(f_{\eta}\right) \\
& +3 \cdot G_{2 f f^{\prime}} \cdot\left(f_{\eta \eta}\right)^{\prime}\left(f_{\eta}\right)^{\prime}+G_{2 f^{\prime}} \cdot\left(f_{\eta \eta \eta}\right)^{\prime}+G_{2 \phi^{\prime \prime} \phi^{\prime \prime} \phi^{\prime \prime}} \cdot\left(\phi_{\eta}\right)^{\prime \prime 2} \\
& +3 \cdot G_{2 \phi^{\prime \prime} \phi^{\prime \prime} f} \cdot\left(\phi_{\eta}\right)^{\prime \prime 2} \cdot\left(f_{\eta}\right)+3 \cdot G_{2 \phi^{\prime \prime} \phi^{\prime \prime} f^{\prime}} \cdot\left(\phi_{\eta}\right)^{\prime \prime 2} \cdot\left(f_{\eta}\right)^{\prime}+3 \cdot G_{2 \phi^{\prime \prime} \phi^{\prime \prime}} \cdot\left(\phi_{\eta}\right)^{\prime \prime} \cdot\left(\phi_{\eta \eta}\right)^{\prime \prime} \\
& +3 \cdot G_{\phi^{\prime \prime} f} \cdot\left(\phi_{\eta \eta}\right)^{\prime \prime} \cdot f_{\eta}+3 \cdot G_{\phi^{\prime \prime} f^{\prime}} \cdot\left(\phi_{\eta \eta}\right)^{\prime \prime} \cdot\left(f_{\eta}\right)^{\prime}+4 \cdot G_{2 \phi^{\prime \prime} f f^{\prime}} \cdot\left(\phi_{\eta}\right)^{\prime \prime} \cdot f_{\eta} \cdot\left(f_{\eta}\right)^{\prime} \\
& +2 \cdot G_{\phi^{\prime \prime} f f} \cdot\left(\phi_{\eta}\right)^{\prime \prime} \cdot\left(f_{\eta}\right)^{2}+2 \cdot G_{\phi^{\prime \prime} f f^{\prime}} \cdot\left(\phi_{\eta}\right)^{\prime \prime} \cdot\left(f_{\eta}\right)^{\prime 2}+2 \cdot G_{2 \phi^{\prime \prime} f} \cdot\left(\phi_{\eta}\right)^{\prime \prime} \cdot f_{\eta \eta} \\
& +2 \cdot G_{2 \phi^{\prime \prime} f^{\prime}} \cdot\left(\phi_{\eta}\right)^{\prime \prime} \cdot\left(f_{\eta \eta}\right)^{\prime}+G_{2 \phi^{\prime \prime}} \cdot\left(\phi_{\eta \eta \eta}\right)^{\prime \prime} \text {, }
\end{aligned}
$$




$$
\begin{aligned}
& G_{3}^{\prime \prime \prime}[\phi(\eta)]=\frac{\mathrm{d}^{3} G_{3}[\phi(\eta)]}{\mathrm{d} \eta^{3}} \\
& =G_{3 \beta^{\prime \prime} \beta^{\prime \prime} \beta^{\prime}} \cdot\left(\beta_{\eta}\right)^{\prime \prime 3}+3 \cdot G_{3 \beta^{\prime \prime} \beta^{\prime \prime} \phi} \cdot\left(\beta_{\eta}\right)^{\prime \prime 2} \phi_{\eta}+3 \cdot G_{3 \beta^{\prime \prime} \beta^{\prime \prime}} \cdot\left(\beta_{\eta}\right)^{\prime \prime} \cdot\left(\beta_{\eta \eta}\right)^{\prime \prime} \\
& +G_{3 \phi \phi \phi} \cdot\left(\phi_{\eta}\right)^{3}+3 \cdot G_{3 \phi \phi \beta^{\prime \prime}} \cdot\left(\phi_{\eta}\right)^{2} \cdot \beta_{\eta}^{\prime \prime}+3 \cdot G_{3 \phi \phi} \cdot\left(\phi_{\eta}\right) \phi_{\eta \eta} \\
& +3 \cdot G_{3 \phi \beta} \cdot\left(\phi_{\eta \eta}\right) \cdot \beta_{\eta}+G_{3 \phi} \cdot\left(\phi_{\eta \eta \eta}\right)+3 \cdot G_{3 \beta^{\prime \prime} \phi} \cdot\left(\beta_{\eta \eta}\right)^{\prime \prime} \cdot \phi_{\eta}+G_{3 \beta^{\prime \prime}} \cdot\left(\beta_{\eta \eta \eta}\right)^{\prime \prime} .
\end{aligned}
$$

We note that the derivatives of $f$ with respect $\eta$ that are given in (19), can be computing by Equations (35)-(38) as

$$
\begin{aligned}
& G_{1}\left[f_{0}(\eta)\right]=-2 \alpha \operatorname{Ref}_{0}(\eta) f_{0}^{\prime}(\eta)-(4-H a) \alpha^{2} f_{0}^{\prime}(\eta), \\
& G_{2}\left[\beta_{0}(\eta)\right]=-E_{c} P_{r}\left[4 \alpha^{2} f_{0}^{2}(\eta)-\left(f_{0}^{\prime}(\eta)\right)^{2}\right]-D_{f} P_{r} \phi_{0}^{\prime \prime}(\eta), \\
& G_{3}\left[\phi_{0}(\eta)\right]=-S_{c} S_{r} \beta_{0}^{\prime \prime}+S_{c} \gamma \alpha^{2} \phi_{0}(\eta), \\
& G_{1}^{\prime}\left[f_{0}(\eta)\right]=G_{1 f_{0}} \cdot f_{1}+G_{1 f_{0}^{\prime}} \cdot\left(f_{1}\right)^{\prime}, \\
& G_{2}^{\prime}\left[\beta_{0}(\eta)\right]=G_{2 f_{0}} \cdot f_{1}+G_{2 f_{0}^{\prime}} \cdot\left(f_{1}\right)^{\prime}+G_{2 \phi_{0}^{\prime \prime}} \cdot\left(\phi_{1}\right)^{\prime \prime} \text {, } \\
& G_{3}^{\prime}\left[\phi_{0}(\eta)\right]=G_{3 \beta_{0}^{\prime \prime}} \cdot\left(\beta_{1}\right)^{\prime \prime}+G_{3 \phi_{0}} \cdot\left(\phi_{1}\right), \\
& G_{1}^{\prime \prime}\left[f_{0}(\eta)\right]=G_{f_{0} f_{0}} \cdot\left(f_{1}\right)^{2}+2 \cdot G_{f_{0} f_{0}^{\prime}} \cdot f_{1}\left(f_{1}\right)^{\prime}+G_{f_{0}^{\prime} f_{0}^{\prime}} \cdot\left(f_{1}\right)^{\prime 2} \\
& +G_{f_{0}} \cdot f_{2}+G_{f_{0}^{\prime}} \cdot\left(f_{2}\right)^{\prime} \\
& G_{2}^{\prime \prime}\left[\beta_{0}(\eta)\right]=G_{f_{0} f_{0}} \cdot\left(f_{1}\right)^{2}+2 \cdot G_{f_{0} f_{0}^{\prime}} \cdot f_{1}\left(f_{1}\right)^{\prime}+G_{f_{0}^{\prime} f_{0}^{\prime}} \cdot\left(f_{1}\right)^{\prime 2}+G_{f_{0}} \cdot f_{2} \\
& +G_{f_{0}^{\prime}} \cdot\left(f_{2}\right)^{\prime}+G_{2 \phi_{0}^{\prime \prime} \phi_{0}^{n}} \cdot\left(\phi_{1}\right)^{\prime \prime 2}+2 \cdot G_{2 \phi_{0}^{\prime \prime} f_{0}} \cdot\left(\phi_{1}\right)^{\prime \prime} \cdot f_{1} \\
& +2 \cdot G_{2 \phi_{0}^{\prime \prime} f_{0}^{\prime}} \cdot\left(\phi_{1}\right)^{\prime \prime} \cdot\left(f_{1}\right)^{\prime}+G_{2 \phi_{0}^{\prime \prime}} \cdot\left(\phi_{2}\right)^{\prime \prime} \\
& G_{3}^{\prime \prime}\left[\phi_{0}(\eta)\right]=G_{3 \beta_{0}^{\prime \prime} \beta_{0}^{\prime \prime}} \cdot\left(\beta_{1}\right)^{\prime \prime 2}+G_{3 \phi_{0} \phi_{0}} \cdot\left(\phi_{1}\right)^{2}+2 G_{3 \beta_{0}^{\prime \prime} \phi_{0}} \cdot\left(\beta_{1}\right)^{\prime \prime} \cdot \phi_{1} \\
& +G_{3 \phi_{0}} \cdot\left(\phi_{2}\right)+G_{3 \beta_{0}^{\prime \prime}} \cdot\left(\beta_{2}\right)^{\prime \prime} \\
& G_{1}^{\prime \prime \prime}\left[f_{0}(\eta)\right]=G_{1 f_{0} f_{0} f_{0}}\left(f_{1}\right)^{3}+3 \cdot G_{1 f_{0} f_{0} f_{0}^{\prime}}\left(f_{1}\right)^{2} \cdot\left(f_{1}\right)^{\prime}+3 \cdot G_{1 f_{0} f_{0}^{\prime} f_{0}^{\prime}} \cdot\left(f_{1}\right)\left(f_{1}\right)^{\prime 2} \\
& +G_{1 f_{0}^{\prime} f_{0}^{\prime} f_{0}^{\prime}} \cdot\left(f_{3}\right)^{\prime 3}+3 \cdot G_{1 f_{0} f_{0}} \cdot f_{2} \cdot f_{1}+3 \cdot G_{1 f_{0} f_{0}^{\prime}} \cdot f_{2} \cdot\left(f_{1}\right)^{\prime}+G_{f_{0}} \cdot f_{3} \\
& +3 \cdot G_{1 f_{0}^{\prime} f_{0}} \cdot\left(f_{2}\right)^{\prime}\left(f_{1}\right)+3 \cdot G_{1 f_{0}^{\prime} f_{0}^{\prime}} \cdot\left(f_{2}\right)^{\prime}\left(f_{1}\right)^{\prime}+G_{1 f_{0}^{\prime}} \cdot\left(f_{3}\right)^{\prime} \text {, } \\
& G_{2}^{\prime \prime \prime}\left[\beta_{0}(\eta)\right]=G_{2 f_{0} f_{0} f_{0}}\left(f_{1}\right)^{3}+3 \cdot G_{2 f_{0} f_{0} f_{0}^{\prime}}\left(f_{1}\right)^{2} \cdot\left(f_{1}\right)^{\prime}+3 \cdot G_{2 f_{0} f_{0}^{\prime} f_{0}} \cdot\left(f_{1}\right)\left(f_{1}\right)^{\prime 2} \\
& +G_{2 f_{0}^{\prime} f_{0}^{\prime} f_{0}^{\prime}} \cdot\left(f_{1}\right)^{\prime 3}+3 \cdot G_{2 f_{0} f_{0}} \cdot f_{2} \cdot f_{1}+3 \cdot G_{2 f_{0} f_{0}^{\prime}} \cdot f_{2} \cdot\left(f_{1}\right)^{\prime} \\
& +G_{2 f} \cdot f_{3}+3 \cdot G_{2 f_{0}^{\prime} f_{0}} \cdot\left(f_{2}\right)^{\prime}\left(f_{1}\right)+3 \cdot G_{2 f_{0}^{\prime} f_{0}^{\prime}} \cdot\left(f_{2}\right)^{\prime}\left(f_{1}\right)^{\prime} \\
& +G_{2 f_{0}^{\prime}} \cdot\left(f_{3}\right)^{\prime}+G_{2 \phi_{0}^{\prime \prime} \phi_{0}^{\prime \prime} \phi_{0}^{\prime \prime}} \cdot\left(\phi_{1}\right)^{\prime \prime 2}+3 \cdot G_{2 \phi_{0}^{\prime \prime} \phi_{0}^{\prime \prime} f_{0}} \cdot\left(\phi_{1}\right)^{\prime \prime 2} \cdot\left(f_{1}\right) \\
& +3 \cdot G_{2 \phi_{0}^{\prime \prime} \phi_{0}^{\prime \prime} f_{0}} \cdot\left(\phi_{1}\right)^{\prime \prime 2} \cdot\left(f_{1}\right)^{\prime}+3 \cdot G_{2 \phi_{0}^{\prime \prime} \phi_{0}^{\prime \prime}} \cdot\left(\phi_{1}\right)^{\prime \prime} \cdot\left(\phi_{2}\right)^{\prime \prime}
\end{aligned}
$$




$$
\begin{aligned}
& +3 \cdot G_{\phi_{0}^{\prime \prime} f_{0}} \cdot\left(\phi_{2}\right)^{\prime \prime} \cdot f_{1}+3 \cdot G_{\phi_{0}^{\prime \prime} f_{0}} \cdot\left(\phi_{2}\right)^{\prime \prime} \cdot\left(f_{1}\right)^{\prime} \\
& +4 \cdot G_{2 \phi_{0}^{\prime} f_{0} f_{0}^{\prime}} \cdot\left(\phi_{1}\right)^{\prime \prime} \cdot f_{1} \cdot\left(f_{1}\right)^{\prime}+2 \cdot G_{\phi_{0}^{\prime \prime} f_{0} f_{0}} \cdot\left(\phi_{1}\right)^{\prime \prime} \cdot\left(f_{1}\right)^{2} \\
& +2 \cdot G_{\phi_{0}^{\prime \prime} f_{0}^{\prime} f_{0}^{\prime}} \cdot\left(\phi_{1}\right)^{\prime \prime} \cdot\left(f_{1}\right)^{\prime 2}+2 \cdot G_{2 \phi_{0}^{\prime \prime} f_{0}} \cdot\left(\phi_{1}\right)^{\prime \prime} \cdot f_{2} \\
& +2 \cdot G_{2 \phi_{0}^{\prime \prime} f_{0}^{\prime}} \cdot\left(\phi_{1}\right)^{\prime \prime} \cdot\left(f_{2}\right)^{\prime}+G_{2 \phi_{0}^{\prime \prime}} \cdot\left(\phi_{3}\right)^{\prime \prime}, \\
G_{3}^{\prime \prime \prime}\left[\phi_{0}(\eta)\right]= & G_{3 \beta_{0}^{\prime \prime} \beta_{0}^{\prime \prime} \beta_{0}^{\prime \prime}} \cdot\left(\beta_{1}\right)^{\prime \prime 3}+3 \cdot G_{3 \beta_{0}^{n} \beta_{0}^{\prime \prime} \phi_{0}} \cdot\left(\beta_{1}\right)^{\prime \prime 2} \phi_{1}+3 \cdot G_{3 \beta_{0}^{\prime \prime} \beta_{0}^{n}} \cdot\left(\beta_{1}\right)^{\prime \prime} \cdot\left(\beta_{2}\right)^{\prime \prime} \\
& +G_{3 \phi_{0} \phi_{0} \phi_{0}} \cdot\left(\phi_{1}\right)^{3}+3 \cdot G_{3 \phi_{0} \phi_{0} \beta_{0}^{\prime \prime}} \cdot\left(\phi_{1}\right)^{2} \cdot \beta_{1}^{\prime \prime}+3 \cdot G_{3 \phi_{0} \phi_{0}} \cdot\left(\phi_{1}\right) \phi_{2} \\
& +3 \cdot G_{3 \phi_{0} \beta_{0}} \cdot\left(\phi_{2}\right) \cdot \beta_{1}+G_{3 \phi_{0}} \cdot \phi_{3}+3 \cdot G_{3 \beta_{0}^{\prime \prime} \phi_{0}} \cdot\left(\beta_{2}\right)^{\prime \prime} \cdot \phi_{1}+G_{3 \beta_{0}^{n}} \cdot\left(\beta_{3}\right)^{\prime \prime} .
\end{aligned}
$$

The extraction of the first derivatives of $G$ can be represented as:

$$
\begin{gathered}
G_{1 f_{0}}=-2 \alpha \operatorname{Re} f_{0}^{\prime}(\eta), \quad G_{f_{0} f_{0}}=0, \quad G_{f_{0} f_{0}^{\prime}}=-2 \alpha R e, \\
G_{1 f_{0} f_{0} f_{0}}=G_{1 f_{0} f_{0}^{\prime} f_{0}}=G_{1 f_{0} f_{0}^{\prime} f_{0}^{\prime}}=G_{1 f_{0} f_{0} f_{0}^{\prime}}=0, \\
G_{1 f_{0}^{\prime}}=-2 \alpha \operatorname{Re} f_{0}(\eta)-(4-H a) \alpha^{2}, \quad G_{1 f_{0}^{\prime} f_{0}}=-2 \alpha R e, \quad G_{1 f_{0}^{\prime} f_{0}^{\prime}}=0, \\
G_{1 f_{0}^{\prime} f_{0} f_{0}}=G_{1 f_{0}^{\prime} f_{0}^{\prime} f_{0}}=G_{1 f_{0}^{\prime} f_{0} f_{0}^{\prime}}=G_{1 f_{0}^{\prime} f_{0}^{\prime} f_{0}^{\prime}}=0, \\
G_{2 f_{0}}=-8 E_{c} P_{r} \alpha^{2} f_{0}(\eta), \quad G_{2 f_{0} f_{0}}=-8 E_{c} P_{r} \alpha^{2}, \quad G_{2 f_{0} f_{0}^{\prime}}=0, \\
G_{2 f_{0} f_{0} f_{0}}=G_{2 f_{0} f_{0}^{\prime} f_{0}}=G_{2 f_{0} f_{0}^{\prime} f_{0}^{\prime}}=G_{2 f_{0} f_{0} f_{0}^{\prime}}=0, G_{2 \phi_{0}^{\prime \prime}}=-D_{f} P_{r}, \\
G_{2 f_{0}^{\prime}}=-8 E_{c} P_{r} \alpha^{2} f_{0}^{\prime}(\eta), \quad G_{f_{0}^{\prime} f_{0}}=0, \quad G_{f_{0}^{\prime} f_{0}^{\prime}}=-8 E_{c} P_{r} \alpha^{2}, \\
G_{f_{0}^{\prime} f_{0}}=G_{f_{0}^{\prime} f_{0}^{\prime} f_{0}}=G_{f_{0}^{\prime} f_{0} f_{0}^{\prime}}=G_{f_{0}^{\prime} f_{0}^{\prime} f_{0}^{\prime}}=0, G_{2 \phi_{0}^{\prime \prime} f_{0}}=0, G_{2 \phi_{0}^{\prime \prime} \phi_{0}^{\prime \prime}}=0 \\
G_{\beta_{0}^{\prime \prime}}=-S_{c} S_{r}, \quad G_{\beta_{0}^{\prime \prime} \beta_{0}^{\prime \prime}}=0, \quad G_{\beta_{0}^{\prime \prime} \phi_{0}}=0, G_{\phi_{0}}=S_{c} \gamma \alpha^{2}, \\
G_{\phi_{0} \phi_{0}}=0, G_{\phi_{0} \phi_{0} \phi_{0}}=G_{\beta_{0}^{\prime \prime} \phi_{0} \phi_{0}}=G_{\phi_{0} \beta_{0}^{\prime \prime} \phi_{0}}=G_{\phi_{0} \phi_{0} \beta_{0}^{\prime \prime}}=0,
\end{gathered}
$$

from Equation (33) by using Equations (39)-(42), gives the following,

$$
\begin{aligned}
& f_{0}=\frac{1}{2} A_{3} \eta^{2}+1 \\
& \beta_{0}=B_{1}, \\
& \phi_{0}=C_{1} \text {, } \\
& f_{1}=-\frac{1}{120} \alpha \operatorname{Re} A_{3}^{2} \eta^{6}-\left(\frac{1}{12} \alpha \operatorname{Re} A_{3}+\frac{1}{6} \alpha^{2} A_{3}-\frac{1}{24} \alpha^{2} \mathrm{HaA}_{3}\right) \eta^{4}, \\
& \beta_{1}=-\frac{1}{30} \alpha^{2} P_{r} E_{c} A_{3}^{2} \eta^{6}-\frac{1}{12}\left(4 A_{3} \alpha^{2}+A_{3}^{2}\right) P_{r} E_{c} \eta^{4}-2 \alpha^{2} P_{r} E_{c} \eta^{2}, \\
& \phi_{1}=\frac{1}{2} \gamma \alpha^{2} S_{c} C_{1} \text {, } \\
& f_{2}=\frac{1}{10800} \alpha^{2} \operatorname{Re}^{2} A_{3}^{3} \eta^{10}+\left(\frac{1}{280} \alpha^{3} R e A_{3}^{2}-\frac{1}{1120} \alpha^{3} \operatorname{ReHaA}_{3}^{2}+\frac{1}{560} \alpha^{2} \operatorname{Re}^{2} A_{3}^{2}\right) \eta^{8} \\
& +\left(\frac{1}{180} \alpha^{2} \operatorname{Re}^{2} A_{3}+\frac{1}{45} \alpha^{3} \operatorname{ReA}_{3}-\frac{1}{180} \alpha^{3} \mathrm{ReHaA}_{3}\right. \\
& \left.+\frac{1}{45} \alpha^{4} A_{3}+\frac{1}{90} \alpha^{4} \mathrm{ReHaA}_{3}+\frac{1}{720} \alpha^{4} \mathrm{ReHa}^{2} A_{3}\right) \eta^{6},
\end{aligned}
$$




$$
\begin{aligned}
& \beta_{2}=\frac{1}{2700} \alpha^{2} \operatorname{Re}^{2} A_{3}^{3} P_{r} E_{c} \eta^{10}+\frac{1}{1680} \alpha A_{3}^{2} P_{r} E_{c}\left(-5 H a \alpha^{3}+12 \alpha^{2} \operatorname{Re}\right. \\
& \left.+20 \alpha^{3}+3 A_{3} R e\right) \eta^{4}+2 S_{r} S_{c} P-r E_{c} \alpha^{2} \eta^{2}, \\
& \phi_{2}=\frac{1}{30} \alpha^{2} P_{r} E_{c} S_{r} S_{c} A_{3}^{2} \eta^{6}+\left(\frac{1}{12} P_{r} E_{c} S_{r} S_{c}\left(4 A_{3} \alpha^{2}+A_{3}^{2}\right)+\frac{1}{24} S_{c}^{2} \gamma^{2} \alpha^{4} C_{1}\right) \eta^{4} \text {, } \\
& f_{3}=-\frac{1}{1572480} \alpha^{3} \operatorname{Re}^{3} A_{3}^{4} \eta^{14}-\left(\frac{359}{9979200} \alpha^{4} \operatorname{Re}^{2} A_{3}^{3}-\frac{359}{39916800} \alpha^{4} \operatorname{Re}^{2} \mathrm{HaA}_{3}^{3}\right. \\
& \left.+\frac{359}{19958400} \alpha^{3} \operatorname{Re}^{3} A_{3}^{3}\right) \eta^{12}+\left(\frac{29}{226800} \alpha^{4} \operatorname{Re}^{2} \mathrm{HaA}_{3}^{2}+\frac{29}{113400} \alpha^{5} \mathrm{ReHaA}_{3}^{2}\right. \\
& -\frac{29}{907200} \alpha^{5} \mathrm{ReHa}^{2} A_{3}^{2}-\frac{29}{56700} \alpha^{4} \mathrm{Re}^{2} A_{3}^{2}-\frac{29}{226800} \alpha^{3} \operatorname{Re}^{3} A_{3}^{2} \\
& \left.-\frac{29}{56700} \alpha^{5} R e A_{3}^{2}\right) \eta^{10}-\left(\frac{1}{10080} \alpha^{3} \operatorname{Re}^{3} A_{3}+\frac{1}{1680} \alpha^{4} \operatorname{Re}^{2} A_{3}\right. \\
& -\frac{1}{6720} \alpha^{4} \mathrm{Re}^{2} \mathrm{HaA}_{3}+\frac{1}{840} \alpha^{5} \mathrm{ReA}_{3}-\frac{1}{1680} \alpha^{5} \mathrm{ReHaA}_{3} \\
& +\frac{1}{13440} \alpha^{5} \mathrm{ReHa}^{2} \mathrm{~A}_{3}+\frac{1}{1260} \alpha^{6} \mathrm{~A}_{3}-\frac{1}{1680} \alpha^{6} \mathrm{HaA}_{3} \\
& \left.+\frac{1}{6720} \alpha^{6} H a^{2} A_{3}-\frac{1}{80640} \alpha^{6} H a^{3} A_{3}\right) \eta^{8}, \\
& \beta_{3}=-\frac{1}{393120} P_{r} E_{c} \alpha^{4} \operatorname{Re}^{2} A_{3}^{4} \eta^{14}-\frac{1}{9979200} P_{r} E_{c} A_{3}^{3} \alpha^{2} \operatorname{Re}\left(-345 H a \alpha^{3}\right. \\
& \left.+718 R e \alpha^{2}+1380 \alpha^{3}+259 A_{3} R e\right) \eta^{12}+\frac{1}{453600} P_{r} E_{c} A_{3}^{2} \alpha^{2}\left(-49 H a^{2} \alpha^{4}\right. \\
& +214 \text { HaRe }^{3}+392 \mathrm{Ha \alpha}^{4}+120 \mathrm{~A}_{3} \text { HaRe } \alpha-232 \operatorname{Re}^{2} \alpha^{2}-856 \operatorname{Re}^{3} \\
& \left.-784 \alpha^{4}-240 A_{3} \operatorname{Re}^{2}-480 A_{3} \operatorname{Re} \alpha\right) \eta^{10}-\frac{1}{20160} P_{r} E_{c} A_{3} \alpha^{3}\left(2 H a^{2} \alpha^{4}\right. \\
& +13 A_{3} H^{2} \alpha^{2}-8 H a R e \alpha^{3}-16 H_{a \alpha} \alpha^{4}-52 A_{3} \text { HaRe } \alpha-104 A_{3} H_{a \alpha}{ }^{2} \\
& \left.+8 R E^{2} \alpha^{2}+32 R e \alpha^{3}+32 \alpha^{4}+52 A_{3} R E^{2}+208 A_{3} \operatorname{Re} \alpha+208 A_{3} \alpha^{2}\right) \eta^{8} \\
& -\frac{1}{60} P_{r}^{2} D_{f} S_{r} S_{c} E_{c} \alpha^{2} A_{3}^{2} \eta^{6}-\frac{1}{24} P_{r} D_{f}\left(S_{r} S_{c} P_{r} E_{c} A_{3}\left(4 \alpha^{2}+A_{3}\right)\right. \\
& \left.+\frac{1}{2} S_{2} \gamma^{2} \alpha^{4} C_{1}\right) \eta^{4}-\operatorname{Pr}^{2} D_{f} S_{r} S_{c} E_{c} \alpha^{2} \eta^{2} \\
& \phi_{3}=-\frac{1}{5400} S_{r} S_{c} P_{r} E_{c} \alpha^{3} \operatorname{ReA}_{3}^{3} \eta^{10}+\frac{1}{16}\left(-\frac{1}{210} S_{r} S_{c} P_{r} E_{c} \alpha A_{3}^{2}\left(-5 H a \alpha^{3}\right.\right. \\
& \left.\left.+12 R e \alpha^{2}+20 \alpha^{3}+3 A_{3} \operatorname{Re}\right)+a \frac{1}{210} S_{c}^{2} \gamma \alpha^{4} S_{r} P_{r} E_{c} A_{3}^{2}\right) \eta^{8} \\
& +\frac{1}{12}\left(\frac{1}{15} S_{r} S_{c} P_{r} E_{c} \alpha A_{3}\left(H a \alpha^{3}+A_{3} H a \alpha-2 R e \alpha^{2}-4 \alpha^{3}-2 A_{3} \alpha\right)\right. \\
& \left.+\frac{1}{5} S_{c} \gamma \alpha^{2}\left(\frac{1}{12} S_{r} S_{c} P_{r} A_{3}\left(4 \alpha^{2}+A_{3}\right)+\frac{1}{24} S_{c}^{2} \gamma^{2} \alpha^{4} C_{1}\right)\right) \eta^{6} \\
& +\frac{1}{12} S_{c}^{2} \gamma S-r P_{r} E_{c} \alpha^{4} \eta^{4}+\frac{1}{4} S_{r} S_{c} P_{r} D_{f} \gamma \alpha^{2} \operatorname{Re} C_{1} \eta^{2}
\end{aligned}
$$


From step (4) substitution Equations (46)-(42) in Equation (34), the analytical-approximate solution can be resulted as follows:

$$
\begin{aligned}
& f(\eta)=1+\frac{1}{2} A_{3} \eta^{2}-\left(\frac{1}{12} \alpha R e A_{3}+\frac{1}{6} \alpha^{2} A_{3}-\frac{1}{24} \alpha^{2} H a A_{3}\right) \eta^{4}+\left(\frac{1}{180} \alpha^{2} R e^{2} A_{3}\right. \\
& -\frac{1}{120} \alpha \operatorname{ReA}_{3}^{2}+\frac{1}{45} \alpha^{3} \mathrm{ReA}_{3}-\frac{1}{180} \alpha^{3} \mathrm{ReHaA}_{3}+\frac{1}{45} \alpha^{4} A_{3}+\frac{1}{90} \alpha^{4} \mathrm{ReHaA}_{3} \\
& \left.+\frac{1}{720} \alpha^{4}+\mathrm{ReHa}^{2} A_{3}\right) \eta^{6}+\left(\frac{1}{280} \alpha^{3} \mathrm{ReA}_{3}^{2}-\frac{1}{1120} \alpha^{3} \mathrm{ReHaA}_{3}^{2}\right. \\
& +\frac{1}{560} \alpha^{2} R e^{2} A_{3}^{2}-\left(\frac{1}{10080} \alpha^{3} R e^{3} A_{3}+\frac{1}{1680} \alpha^{4} \operatorname{Re}^{2} A_{3}-\frac{1}{6720} \alpha^{4} R e^{2} H_{a} A_{3}\right. \\
& +\frac{1}{840} \alpha^{5} \mathrm{ReA}_{3}-\frac{1}{1680} \alpha^{5} \mathrm{ReHaA}_{3}+\frac{1}{13440} \alpha^{5} \mathrm{ReHa}^{2} A_{3}+\frac{1}{1260} \alpha^{6} A_{3} \\
& \left.-\frac{1}{1680} \alpha^{6} H a A_{3}+\frac{1}{6720} \alpha^{6} H a^{2} A_{3}-\frac{1}{80640} \alpha^{6} H a^{3} A_{3}\right) \eta^{8} \\
& +\frac{1}{10800} \alpha^{2} \operatorname{Re}^{2} A_{3}^{3} \eta^{10}+\cdots \\
& \beta(\eta)=B_{1}+\left(-2 \alpha^{2} P_{r} E_{c}+2 S_{r} S_{c} P_{r} E_{c} \alpha^{2}\right) \eta^{2}+\left(-\frac{1}{12}\left(4 A_{3} \alpha^{2}+A_{3}^{2}\right) P_{r} E_{c}\right. \\
& \left.+\frac{1}{1680} \alpha A_{3}^{2} P_{r} E_{c}\left(-5 H a \alpha^{3}+12 \alpha^{2} R e+20 \alpha^{3}+3 A_{3} R e\right)\right) \eta^{4} \\
& -\frac{1}{30} \alpha^{2} P_{r} E_{c} A_{3}^{2} \eta^{6}+\frac{1}{2700} \alpha^{2} \operatorname{Re}^{2} A_{3}^{3} P_{r} E_{c} \eta^{10}+\cdots \\
& \phi(\eta)=C_{1}+\frac{1}{2} \gamma \alpha^{2} S_{c} C_{1} \eta^{2}+\left(\frac{1}{12} P_{r} E_{c} S_{r} S_{c}\left(4 A_{3} \alpha^{2}+A_{3}^{2}\right)\right. \\
& \left.+\frac{1}{24} l S_{c}^{2} \gamma^{2} \alpha^{4} C_{1}\right) \eta^{4}+\frac{1}{30} \alpha^{2} P_{r} E_{c} S_{r} S_{c} A_{3}^{2} \eta^{6}+\cdots
\end{aligned}
$$

\section{The Analysis of Convergence}

Here, the analysis of convergence for the analytical-approximate solution (50) that was resulted from the application of new power series algorithm for solving the problem has been extensively studied.

Definition (1): Suppose that $H$ is Banach space, $R$ is the real numbers and $G[F, H, P]=\left(G_{1}[F], G_{2}[H], G_{2}[P]\right)$ is a nonlinear operators defined by $G[F, H, P]: H^{3} \rightarrow R^{3}$. Then the sequence of the solutions generated from a new algorithm can be written as

$$
\begin{aligned}
& F_{n+1}=G_{1}\left[F_{n}\right], \quad F_{n}=\sum_{k=0}^{n} f_{k}, \quad n=0,1,2,3, \cdots \\
& H_{n+1}=G_{2}\left[H_{n}\right], \quad H_{n}=\sum_{k=0}^{n} \beta_{k}, \quad n=0,1,2,3, \cdots \\
& P_{n+1}=G_{3}\left[P_{n}\right], \quad P_{n}=\sum_{k=0}^{n} \phi_{k}, \quad n=0,1,2,3, \cdots
\end{aligned}
$$

Definition (2):1 Suppose that $G[F, H, P]$ satisfies Lipschitz condition such that for $0 \leq \gamma_{1}, \gamma_{2}, \gamma_{3}<1, \gamma_{1}, \gamma_{2}, \gamma_{3} \in R$, we have 


$$
\begin{aligned}
& \left\|G_{1}\left[F_{n}\right]-G_{1}\left[F_{n-1}\right]\right\| \leq \gamma_{1}\left\|F_{n}-F_{n-1}\right\|, \\
& \left\|G_{2}\left[H_{n}\right]-G_{2}\left[H_{n-1}\right]\right\| \leq \gamma_{2}\left\|H_{n}-H_{n-1}\right\|, \\
& \left\|G_{3}\left[P_{n}\right]-G_{3}\left[P_{n-1}\right]\right\| \leq \gamma_{3}\left\|P_{n}-P_{n-1}\right\|,
\end{aligned}
$$

Now, we assume that $G\left[F_{n}, H_{n}, P_{n}\right]=G(n)$ for simplify with $\gamma=\gamma_{1}+\gamma_{2}+\gamma_{3}$, $0 \leq \gamma<1$ yield,

$$
\|G(n)-G(n-1)\| \leq \gamma\left\|\left(F_{n}, H_{n}, P_{n}\right)-\left(F_{n-1}, H_{n-1}, P_{n-1}\right)\right\| .
$$

The sufficient condition for convergent of the series analytical-approximate solutions $F_{n}, H_{n}, P_{n}$ is given in the following theorems.

Theorem (1):2 The series of the analytical-approximate solution $\left\{S_{n}=\left(F_{n}, H_{n}, P_{n}\right)\right\}_{0}^{\infty}$ generated from new algorithm converge if the following condition is satisfied:

$$
\left\|S_{n}-S_{m}\right\| \rightarrow 0, \text { as } m \rightarrow \infty, \text { for } 0 \leq \gamma<1,
$$

Proof. From the above definition, the next equation can be written as

$$
\begin{aligned}
\left\|S_{n}-S_{m}\right\|= & \left\|\left(F_{n}, H_{n}, P_{n}\right)-\left(F_{m}, H_{m}, P_{m}\right)\right\| \\
= & \left\|\left(\sum_{k=0}^{n} f_{k}, \sum_{k=0}^{n} \beta_{k}, \sum_{k=0}^{n} \phi_{k}\right)-\left(\sum_{k=0}^{m} f_{k}, \sum_{k=0}^{m} \beta_{k}, \sum_{k=0}^{m} \phi_{k}\right)\right\| \\
= & \|\left(f_{0}+L^{-1} \sum_{k=0}^{n} \frac{\Delta^{k}}{k !} \frac{\mathrm{d}^{(k)} G_{1}\left[f_{0}(\eta)\right]}{\mathrm{d} z^{(\eta)}}, \beta_{0}+L^{-1} \sum_{k=0}^{n} \frac{\Delta^{k}}{k !} \frac{\mathrm{d}^{(k)} G_{2}\left[\beta_{0}(\eta)\right]}{\mathrm{d} \eta^{(k)}},\right. \\
& \phi_{0}+L^{-1} \sum_{k=0}^{n} \frac{\Delta^{k}}{k !} \frac{\mathrm{d}^{(k)} G_{3}\left[\phi_{0}(\eta)\right]}{\mathrm{d} \eta^{(k)}}-\left(f_{0}+L^{-1} \sum_{k=0}^{m} \frac{\Delta^{k}}{k !} \frac{\mathrm{d}^{(k)} G_{1}\left[f_{0}(\eta)\right]}{k ! \mathrm{d} \eta^{(k)}}\right. \\
= & \left.\beta_{0}+L^{-1} \sum_{k=0}^{m} \frac{\Delta^{k}}{k !} \frac{\mathrm{d}^{(k)} G_{2}\left[\beta_{0}(\eta)\right]}{k ! \mathrm{d} \eta^{(k)}}, \phi_{0}+L^{-1} \sum_{k=0}^{m} \frac{\Delta^{k}}{k !} \frac{\mathrm{d}^{(k)} G_{3}\left[\phi_{0}(\eta)\right]}{k ! \mathrm{d} \eta^{(k)}}\right) \| \\
\leq & \left.\mid L^{-1} \| \sum_{k=0}^{n-1} f_{k}, \sum_{k=0}^{n-1} \beta_{k}, \sum_{k=0}^{n-1} \phi \sum_{k=0}^{n-1} f_{k}, \sum_{k=0}^{n-1} \beta_{k}, \sum_{k=0}^{n-1} \phi_{k}\right]-G\left[\sum_{k=0}^{-1} G\left[\sum_{k=0}^{m-1} f_{k}, \sum_{k=0}^{m-1} \sum_{k=0}^{m-1} \beta_{k}, \sum_{k=0}^{m-1} \phi_{k=0}^{m-1} \phi_{k}\right] \|\right. \\
\leq & \mid L^{-1}\left\|G\left[F_{n-1}, H_{n-1}, P_{n-1}\right]-G\left[F_{m-1}, H_{m-1}, P_{m-1}\right]\right\| \\
\leq & \gamma\left\|\left(F_{n-1}, H_{n-1}, P_{n-1}\right)-\left(F_{m-1}, H_{m-1}, P_{m-1}\right)\right\| \\
= & \gamma\left\|S_{n-1}-S_{m-1}\right\|,
\end{aligned}
$$

since $\mathrm{G}[\mathrm{F}, \mathrm{H}, \mathrm{P}]$ satisfies Lipschitz condition. Let $n=m+1$, then

$$
\begin{aligned}
& \left\|F_{m+1}-F_{m}\right\| \leq \gamma_{1}\left\|F_{m}-F_{m-1}\right\|, \\
& \left\|H_{m+1}-H_{m}\right\| \leq \gamma_{2}\left\|H_{m}-H_{m-1}\right\|, \\
& \left\|P_{m+1}-P_{m}\right\| \leq \gamma_{3}\left\|P_{m}-P_{m-1}\right\|,
\end{aligned}
$$

hence,

$$
\begin{aligned}
& \left\|F_{m}-F_{m-1}\right\| \leq \gamma_{1}\left\|F_{m-1}-F_{m-2}\right\| \leq \cdots \leq \gamma_{1}^{m-1}\left\|F_{1}-F_{0}\right\|, \\
& \left\|H_{m}-H_{m-1}\right\| \leq \gamma_{2}\left\|H_{m-1}-H_{m-2}\right\| \leq \cdots \leq \gamma_{2}^{m-1}\left\|H_{1}-H_{0}\right\|, \\
& \left\|P_{m}-P_{m-1}\right\| \leq \gamma_{3}\left\|P_{m-1}-P_{m-2}\right\| \leq \cdots \leq \gamma_{3}^{m-1} \leq\left\|P_{1}-P_{0}\right\|,
\end{aligned}
$$


from Equation (57) we get

$$
\begin{aligned}
& \left\|F_{2}-F_{1}\right\| \leq \gamma_{1}\left\|F_{1}-F_{0}\right\|,\left\|H_{2}-H_{1}\right\| \leq \gamma_{2}\left\|H_{1}-H_{0}\right\|,\left\|P_{2}-P_{1}\right\| \leq \gamma_{3}\left\|P_{1}-P_{0}\right\|, \\
& \left\|F_{3}-F_{2}\right\| \leq \gamma_{1}^{2}\left\|F_{1}-F_{0}\right\|,\left\|H_{3}-H_{2}\right\| \leq \gamma_{2}^{2}\left\|H_{1}-H_{0}\right\|,\left\|F_{3}-F_{2}\right\| \leq \gamma_{3}^{2}\left\|F_{1}-F_{0}\right\|, \\
& \left\|F_{4}-F_{3}\right\| \leq \gamma_{1}^{3}\left\|F_{1}-F_{0}\right\|,\left\|H_{4}-H_{3}\right\| \leq \gamma_{2}^{3}\left\|H_{1}-H_{0}\right\|,\left\|P_{4}-P_{3}\right\| \leq \gamma_{3}^{3}\left\|P_{1}-P_{0}\right\| \\
& \quad \quad \vdots \\
& \left\|F_{m}-F_{m-1}\right\| \leq \gamma_{1}^{m-1}\left\|F_{1}-F_{0}\right\|,\left\|H_{m}-H_{m-1}\right\| \leq \gamma_{2}^{m-1} \leq\left\|H_{1}-H_{0}\right\|, \\
& \left\|P_{m}-P_{m-1}\right\| \leq \gamma_{3}^{m-1}\left\|P_{1}-P_{0}\right\|
\end{aligned}
$$

By using triangle inequality, we find that as $m \rightarrow \infty$, we have $\left\|S_{n}-S_{m}\right\| \rightarrow 0$, then $S_{n}$ is a Cauchy sequence in Banach space $H^{\hat{p}}$.

Theorem (2):3 Let $G=\left(G_{1}, G_{2}, G_{3}\right)$ be a nonlinear operator satisfies Lipschitz condition from $H^{\hat{\beta}}$ to $H^{\hat{\beta}}$. If the series analytical-approximate solution $\left\{S_{n}\right\}$ converges, then it is converged to the solution of the problem (9)-(10).

Proof.

$$
\begin{aligned}
& \left\|G\left[S_{2}\right]-G\left[S_{1}\right]\right\| \\
& =\left\|\left(G_{1}\left[F_{2}\right], G_{2}\left[H_{2}\right], G_{3}\left[P_{2}\right]\right)-\left(G_{1}\left[F_{1}\right], G_{2}\left[H_{1}\right], G_{3}\left[P_{1}\right]\right)\right\| \\
& =\left\|G_{1}\left[F_{2}\right]-G_{1}\left[F_{1}\right], G_{2}\left[H_{2}\right]-G_{2}\left[H_{1}\right], G_{3}\left[P_{2}\right]-G_{3}\left[P_{1}\right]\right\| \\
& =\left\|G_{1}\left[F_{2}\right]-G_{1}\left[F_{1}\right]\right\|+\left\|G_{2}\left[H_{2}\right]-G_{2}\left[H_{1}\right]\right\|+\left\|G_{3}\left[P_{2}\right]-G_{3}\left[P_{1}\right]\right\| \\
& \quad \leq \gamma_{1}\left\|F_{2}-F_{1}\right\|+\gamma_{2}\left\|H_{2}-H_{1}\right\|+\gamma_{3}\left\|P_{2}-P_{1}\right\| \\
& \leq\left(\gamma_{1}+\gamma_{2}+\gamma_{3}\right)\left\|\left(F_{2}, H_{2}, P_{2}\right)-\left(F_{1}, H_{1}, P_{1}\right)\right\| \\
& =\gamma\left\|S_{2}-S_{1}\right\|
\end{aligned}
$$

Therefore, from the Banach fixed-point theorem, there is a unique solution of the problem (9)-(10). We will prove that $\left\{S_{n}\right\}_{0}^{\infty}$ converges to $S$.

$$
G[S]=G\left[\sum_{k=0}^{\infty} S_{k}\right]=\lim _{n \rightarrow \infty} G\left[\sum_{k=0}^{n} S_{k}\right]=\lim _{n \rightarrow \infty} G\left[S_{n}\right]=\lim _{n \rightarrow \infty} S_{n+1}=S .
$$

In practice, the theorems (1) and (2) suggest to compute the value of $\gamma_{1}, \gamma_{2}, \gamma_{3}$, as described in the following definition.

Definition (1):4 for $k=1,2,3, \cdots$

$$
\begin{gathered}
\gamma_{1}^{k}= \begin{cases}\frac{\left\|F_{k+1}-F_{k}\right\|}{\left\|F_{1}-F_{0}\right\|}=\frac{\left\|f_{k+1}\right\|}{\left\|f_{1}\right\|}, & \left\|f_{1}\right\| \neq 0, \\
0, & \left\|f_{1}\right\|=0,\end{cases} \\
\gamma_{2}^{k}= \begin{cases}\frac{\left\|H_{k+1}-H_{k}\right\|}{\left\|H_{1}-H_{0}\right\|}=\frac{\left\|\beta_{k+1}\right\|}{\left\|\beta_{1}\right\|}, & \left\|\beta_{1}\right\| \neq 0, \\
0, & \left\|\beta_{1}\right\|=0,\end{cases} \\
\gamma_{3}^{k}= \begin{cases}\frac{\left\|P_{k+1}-P_{k}\right\|}{\left\|P_{1}-P_{0}\right\|}=\frac{\left\|\phi_{k+1}\right\|}{\left\|\phi_{1}\right\|}, & \left\|\phi_{1}\right\| \neq 0, \\
0, & \left\|\phi_{1}\right\|=0 .\end{cases}
\end{gathered}
$$


Now, the definition (1) can be applied on the magneto hydrodynamic (MHD) flow of viscous fluid in a channel with non-parallel plates to find convergence, then to obtain for examples as below.

If we choose $R e=30, \alpha=3^{\circ}, H a=500, S_{r}=S_{c}=0.1, \quad P_{r}=0.1$, $D_{f}=0.01, E_{c}=0.01, \gamma=0.04$ then obtain:

$$
\begin{gathered}
\left\|F_{2}-F_{1}\right\|_{2} \leq \gamma_{1}\left\|F_{1}-F_{0}\right\|_{2} \Rightarrow \gamma_{1}=0.08954317<1, \\
\left\|F_{3}-F_{2}\right\|_{2} \leq \gamma_{1}^{2}\left\|F_{1}-F_{0}\right\|_{2} \Rightarrow \gamma_{1}^{2}=0.005099704<1, \\
\left\|F_{4}-F_{3}\right\|_{2} \leq \gamma_{1}^{3}\left\|F_{1}-F_{0}\right\|_{2} \Rightarrow \gamma_{1}^{3}=0.0001956583630<1, \\
\vdots \\
\left\|H_{2}-H_{1}\right\|_{2} \leq \gamma_{2}\left\|H_{1}-H_{0}\right\|_{2} \Rightarrow \gamma_{2}=0.2496673982<1, \\
\left\|H_{3}-H_{2}\right\|_{2} \leq \gamma_{2}^{2}\left\|H_{1}-H_{0}\right\|_{2} \Rightarrow \gamma_{2}^{2}=0.03173895000<1, \\
\left\|H_{4}-H_{3}\right\|_{2} \leq \gamma_{2}^{3}\left\|H_{1}-H_{0}\right\|_{2} \Rightarrow \gamma_{2}^{3}=0.002169482685<1, \\
\vdots \\
\left\|P_{2}-P_{1}\right\|_{2} \leq \gamma_{3}\left\|P_{1}-P_{0}\right\|_{2} \Rightarrow \gamma_{3}=0.73232384<1, \\
\left\|P_{3}-P_{2}\right\|_{2} \leq \gamma_{3}^{2}\left\|P_{1}-P_{0}\right\|_{2} \Rightarrow \gamma_{3}^{2}=0.09141845239<1, \\
\left\|P_{4}-P_{3}\right\|_{2} \leq \gamma_{3}^{3}\left\|P_{1}-P_{0}\right\|_{2} \Rightarrow \gamma_{3}^{3}=0.003873822310<1, \\
\quad \vdots \\
\left\|F_{2}-F_{1}\right\|_{+\infty} \leq \gamma\left\|F_{1}-F_{0}\right\|_{+\infty} \Rightarrow \gamma=0.07399291<1, \\
\left\|F_{3}-F_{2}\right\|_{+\infty} \leq \gamma^{2}\left\|F_{1}-F_{0}\right\|_{+\infty} \Rightarrow \gamma^{2}=0.004719598<1, \\
\left\|F_{4}-F_{3}\right\|_{+\infty} \leq \gamma^{3}\left\|F_{1}-F_{0}\right\|_{+\infty} \Rightarrow \gamma^{3}=0.0001557525428<1, \\
\vdots \\
\left\|H_{2}-H_{1}\right\|_{+\infty} \leq \gamma\left\|H_{1}-H_{0}\right\|_{+\infty} \Rightarrow \gamma=0.2384340096<1, \\
\left\|H_{3}-H_{2}\right\|_{+\infty} \leq \gamma^{2}\left\|H_{1}-H_{0}\right\|_{+\infty} \Rightarrow \gamma^{2}=0.02467876<1, \\
\left\|H_{4}-H_{3}\right\|_{+\infty} \leq \gamma^{3}\left\|H_{1}-H_{0}\right\|_{+\infty} \Rightarrow \gamma^{3}=0.001715583134<1, \\
\vdots \\
\left\|P_{2}-P_{1}\right\|_{+\infty} \leq \gamma\left\|P_{1}-P_{0}\right\|_{+\infty} \Rightarrow \gamma=0.732255116<1, \\
\left\|P_{3}-P_{2}\right\|_{+\infty} \leq \gamma^{2}\left\|P_{1}-P_{0}\right\|_{+\infty} \Rightarrow \gamma^{2}=0.087297019<1, \\
\left\|P_{4}-P_{3}\right\|_{+\infty} \leq \gamma^{3}\left\|P_{1}-P_{0}\right\|_{+\infty} \Rightarrow \gamma^{3}=0.003011824761<1, \\
\vdots
\end{gathered}
$$

Also, if we get $\alpha=-2^{\circ}, R e=10, H a=110, P_{r}=D_{f}=0.2, \gamma=0.4$, $E_{c}=0.01$.

$$
\begin{gathered}
\left\|F_{2}-F_{1}\right\|_{2} \leq \gamma_{1}\left\|F_{1}-F_{0}\right\|_{2} \Rightarrow \gamma_{1}=0.03060256592<1, \\
\left\|F_{3}-F_{2}\right\|_{2} \leq \gamma_{1}^{2}\left\|F_{1}-F_{0}\right\|_{2} \Rightarrow \gamma_{1}^{2}=0.1113608591<1, \\
\left\|F_{4}-F_{3}\right\|_{2} \leq \gamma_{1}^{3}\left\|F_{1}-F_{0}\right\|_{2} \Rightarrow \gamma_{1}^{3}=0.4872382121<1, \\
\vdots \\
\left\|H_{2}-H_{1}\right\|_{2} \leq \gamma_{2}\left\|H_{1}-H_{0}\right\|_{2} \Rightarrow \gamma_{2}=0.0004683459276<1, \\
\left\|H_{3}-H_{2}\right\|_{2} \leq \gamma_{2}^{2}\left\|H_{1}-H_{0}\right\|_{2} \Rightarrow \gamma_{2}^{2}=0.005594660398<1, \\
\left\|H_{4}-H_{3}\right\|_{2} \leq \gamma_{2}^{3}\left\|H_{1}-H_{0}\right\|_{2} \Rightarrow \gamma_{2}^{3}=0.02712979918<1,
\end{gathered}
$$




$$
\begin{gathered}
\left\|P_{2}-P_{1}\right\|_{2} \leq \gamma_{3}\left\|P_{1}-P_{0}\right\|_{2} \Rightarrow \gamma_{3}=0.000005903459683<1, \\
\left\|P_{3}-P_{2}\right\|_{2} \leq \gamma_{3}^{2}\left\|P_{1}-P_{0}\right\|_{2} \Rightarrow \gamma_{3}^{2}=0.0001292820508<1, \\
\left\|P_{4}-P_{3}\right\|_{2} \leq \gamma_{3}^{3}\left\|P_{1}-P_{0}\right\|_{2} \Rightarrow \gamma_{3}^{3}=0.0004542885489<1, \\
\vdots \\
\left\|F_{2}-F_{1}\right\|_{+\infty} \leq \gamma_{1}\left\|F_{1}-F_{0}\right\|_{+\infty} \Rightarrow \gamma_{1}=0.02756015475<1, \\
\left\|F_{3}-F_{2}\right\|_{+\infty} \leq \gamma_{1}^{2}\left\|F_{1}-F_{0}\right\|_{+\infty} \Rightarrow \gamma_{1}^{2}=0.1104543115<1, \\
\left\|F_{4}-F_{3}\right\|_{+\infty} \leq \gamma_{1}^{3}\left\|F_{1}-F_{0}\right\|_{+\infty} \Rightarrow \gamma_{1}^{3}=0.4872217326<1, \\
\quad \vdots \\
\left\|H_{2}-H_{1}\right\|_{+\infty} \leq \gamma_{2}\left\|H_{1}-H_{0}\right\|_{+\infty} \Rightarrow \gamma_{2}=0.00041798914<1, \\
\left\|H_{3}-H_{2}\right\|_{+\infty} \leq \gamma_{2}^{2}\left\|H_{1}-H_{0}\right\|_{+\infty} \Rightarrow \gamma_{2}^{2}=0.005302953669<1, \\
\left\|H_{4}-H_{3}\right\|_{+\infty} \leq \gamma_{2}^{3}\left\|H_{1}-H_{0}\right\|_{+\infty} \Rightarrow \gamma_{2}^{3}=0.02690804181<1, \\
\vdots \\
\left\|P_{2}-P_{1}\right\|_{+\infty} \leq \gamma_{3}\left\|P_{1}-P_{0}\right\|_{+\infty} \Rightarrow \gamma_{3}=0.00000534538<1, \\
\left\|P_{3}-P_{2}\right\|_{+\infty} \leq \gamma_{3}^{2}\left\|P_{1}-P_{0}\right\|_{+\infty} \Rightarrow \gamma_{3}^{2}=0.00010743626<1, \\
\left\|P_{4}-P_{3}\right\|_{+\infty} \leq \gamma_{3}^{3}\left\|P_{1}-P_{0}\right\|_{+\infty} \Rightarrow \gamma_{3}^{3}=0.00043058757<1,
\end{gathered}
$$

Then $\sum_{k=0}^{\infty} f_{k}(\eta), \sum_{k=0}^{\infty} \beta_{k}(\eta)$ and $\sum_{k=0}^{\infty} \phi_{k}(\eta)$ converge to the solutions $f(\eta), \beta(\eta)$ and $\phi(\eta)$ respectively when $0 \leq \gamma_{1}^{k}, \gamma_{2}^{k}, \gamma_{3}^{k}<1, k=1,2, \cdots$.

\section{Results and Discussions}

This section is dedicated to study the influence of various non dimensional physical parameters on velocity field $f(\eta)$, temperature field $\beta(\eta)$ and concentration field $\phi(\eta)$. Also the influence of different parameters on rate of heat transfer and rate of mass transfer are under observation for diverging and converging channels. In Table 1 and Table 2 proof convergence the values $A_{3}, B_{1}$ and $C_{1}$ of initial solutions. The stability of these values can be clearly distinguished from the fourth approximation. Table 3 and Table 4 present a comparison of the solutions obtained by new algorithm and numerical algorithm. These tables show that an excellent between the solutions. On the other hand Tables 5-13 are explained to analyze the behavior of Nusselt number and Sherwood number with variation parameters. As for can say that impotent to mention that Nusselt number gives a description of heat transfer rate at the wall, while Sherwood number represents the rate of mass transfer at the wall. In Table 5 and Table 6, Nusselt number with very Reynolds number Re and channel opening $\alpha$ are discussed. One can clearly observe that for diverging channel, Nusselt number a rise with increasing in Reynolds number, but for increasing Re lead to the rate of heat transfer a drop for converging channel. Sherwood number appears to be increasing as the Reynolds number grows for converging channel and decreases for diverging channel. The behavior of Nusselt number in channel 
opening is quite opposite for Reynolds number. Table 7 gives description of variation in heat transfer rate and mass transfer rate at the wall with the rising values of Hartmman number. Increase in heat transfer rate and mass transfer rate are observed for increasing Hartmman number $\mathrm{Ha}$ in the case of both converging and diverging channels. Table 8 and Table 13 portray that the rate of heat transfer increasing for both diverging and converging channels an increase in Schmidt, Soret, Prandtl, Eckert, Dufour numbers and chemical reaction parameter respectively. Furthermore these tables for both channels clear that the rate of mass transfer at the walls decreases with an increase in Schmidt, Soret, Prandtl, Eckert, Dufour numbers and chemical reaction parameter.

Table 1. $R e=30, H a=500, P_{r}=0.1, D_{f}=0.02, S_{c}=S_{r}=0.1, \gamma=0.04, \alpha=3^{\circ}$.

\begin{tabular}{cccc}
\hline Approximation & $A_{3}$ & $B_{1}$ & $C_{1}$ \\
\hline 1 term & -2.1999729 & 1.00040723 & 0.99999452 \\
2 term & -2.1998869 & 1.00034130 & 0.99999045 \\
3 term & -2.1993584 & 1.00034466 & 0.99999078 \\
4 term & -2.1993648 & 1.00034465 & 0.99999077 \\
5 term & -2.1993648 & 1.00034465 & 0.99999077 \\
6 term & -2.1993648 & 1.00034465 & 0.99999077 \\
7 term & -2.1993648 & 1.00034465 & 0.99999077 \\
8 term & -2.1993648 & 1.00034465 & 0.99999077 \\
\hline
\end{tabular}

Table 2. $R e=10, H a=110, P_{r}=0.2, D_{f}=0.2, S_{c}=S_{r}=0.2, \gamma=0.4, \alpha=-2^{\circ}$.

\begin{tabular}{cccc}
\hline Approximation & $A_{3}$ & $B_{1}$ & $C_{1}$ \\
\hline 1 term & -1.89052474 & 1.00059930 & 0.99995131 \\
2 term & -1.88870911 & 1.00065724 & 0.99992738 \\
3 term & -1.88872478 & 1.00065993 & 0.99992621 \\
4 term & -1.88872513 & 1.00065996 & 0.99992618 \\
5 term & -1.88872513 & 1.00065996 & 0.99992618 \\
6 term & -1.88872513 & 1.00065996 & 0.99992618 \\
7 term & -1.88872513 & 1.00065996 & 0.99992618 \\
\hline
\end{tabular}

Table 3. Comparison between new scheme and $(R-K 4)$ scheme for the analytical solutions $f(\eta), \beta(\eta), \phi(\eta)$ when $R e=30, H a=500, \alpha=3^{\circ}, P_{r}=0.1, \quad S_{c}=S_{r}=0.1$, $E_{c}=0.01, D_{f}=0.04$.

\begin{tabular}{ccccccc}
\hline$\eta$ & $f(\eta)$ & $(R-K 4)$ & $\beta(\eta)$ & $(R-K 4)$ & $\phi(\eta)$ & $\eta$ \\
\hline 0.0 & 1.0000000 & 1.0000000 & 1.00034465 & 1.00034465 & 0.99999077 & 0.99999077 \\
0.1 & 0.9890195 & 0.9890195 & 1.0003445 & 1.0003445 & 0.9999908 & 0.9999908 \\
0.2 & 0.9562698 & 0.9562698 & 1.0003437 & 1.0003437 & 0.9999910 & 0.9999910 \\
\hline
\end{tabular}




\section{Continued}

\begin{tabular}{lllllll}
\hline 0.3 & 0.9022985 & 0.9022985 & 1.0003409 & 1.0003409 & 0.9999913 & 0.9999913 \\
0.4 & 0.8279407 & 0.8279407 & 1.0003338 & 1.0003338 & 0.9999917 & 0.9999917 \\
0.5 & 0.7341800 & 0.7341800 & 1.0003195 & 1.0003195 & 0.9999924 & 0.9999924 \\
0.6 & 0.6220550 & 0.6220550 & 1.0002170 & 1.0002170 & 0.9999932 & 0.9999932 \\
0.7 & 0.4923839 & 0.4923839 & 1.0001958 & 1.0001958 & 0.9999944 & 0.9999944 \\
0.8 & 0.3456799 & 0.3456799 & 1.0001958 & 1.0001958 & 0.9999958 & 0.9999958 \\
0.9 & 0.1818466 & 0.1818466 & 1.0011290 & 1.0011290 & 0.9999977 & 0.9999977 \\
1.0 & 0.0000000 & 0.0000000 & 1.0000000 & 1.0000000 & 1.0000000 & 1.00000000 \\
\hline
\end{tabular}

Table 4. Comparison between new scheme and $(R-K 4)$ scheme for the analytical solutions $f(\eta), \beta(\eta), \phi(\eta)$ when $R e=10, H a=110, P_{r}=0.2, D_{f}=0.2, \quad S_{c}=S_{r}=0.2$, $\gamma=0.4, \alpha=-2^{\circ}$.

\begin{tabular}{ccccccc}
\hline$\eta$ & $f(\eta)$ & $(R-K 4)$ & $\beta(\eta)$ & $(R-K 4)$ & $\phi(\eta)$ & $(R-K 4)$ \\
\hline 0.0 & 1.0000000 & 1.0000000 & 1.0003447 & 1.0003447 & 0.9999908 & 0.9999908 \\
0.1 & 0.9905498 & 0.9905498 & 1.0006599 & 1.0006599 & 0.9999262 & 0.9999262 \\
0.2 & 0.9621219 & 0.9621219 & 1.0006587 & 1.0006587 & 0.9999282 & 0.9999282 \\
0.3 & 0.9144866 & 0.9144866 & 1.0006544 & 1.0006544 & 0.9999308 & 0.9999308 \\
0.4 & 0.8472720 & 0.8472720 & 1.0006434 & 1.0006434 & 0.9999346 & 0.9999346 \\
0.5 & 0.7599801 & 0.7599801 & 1.0006202 & 1.0006202 & 0.9999399 & 0.9999399 \\
0.6 & 0.6520119 & 0.6520119 & 1.0005776 & 1.0005776 & 0.9999469 & 0.9999469 \\
0.7 & 0.52269974 & 0.52269974 & 1.0005067 & 1.0005067 & 0.9999560 & 0.9999560 \\
0.8 & 0.3713515 & 0.3713515 & 1.0003962 & 1.0003962 & 0.9999675 & 0.9999675 \\
0.9 & 0.1973059 & 0.1973059 & 1.0002326 & 1.0002326 & 0.9999820 & 0.9999820 \\
1.0 & 0.0000000 & 0.0000000 & 1.0000000 & 1.0000000 & 1.0000000 & 1.0000000 \\
\hline
\end{tabular}

Table 5. Variation in Nusselt number and Sherwood number with varying $R e$ when $H a=100, E_{c}=1, S_{c}=S_{r}=0.2, D_{f}=P_{r}=0.5, \gamma=0.1$.

\begin{tabular}{ccccc}
\hline & $\alpha=-5^{\circ}$ & \multicolumn{3}{c}{$\alpha=5^{\circ}$} \\
\hline$R e$ & $\alpha N u=\beta^{\prime}(1)$ & $\alpha S h=\phi^{\prime}(1)$ & $\alpha N u=\beta^{\prime}(1)$ & $\alpha S h=\phi^{\prime}(1)$ \\
0 & 0.015725 & -0.025891 & 0.015725 & -0.025890 \\
10 & 0.015501 & -0.023525 & 0.016295 & -0.028446 \\
20 & 0.015403 & -0.021341 & 0.017951 & -0.031205 \\
30 & 0.015357 & -0.019331 & 0.023679 & -0.034198 \\
40 & 0.015334 & -0.017490 & 0.047894 & -0.037470 \\
50 & 0.015300 & -0.015814 & 0.166257 & -0.041098 \\
\hline
\end{tabular}


Table 6. Variation in Nusselt number and Sherwood number with varying $\alpha$ when $R e=25, H a=100, E_{c}=1, S_{c}=S_{r}=0.2, D_{f}=P_{r}=0.5, \gamma=0.1$.

\begin{tabular}{cccccc}
\hline$\alpha$ & $\alpha N u=\beta^{\prime}(1)$ & $\alpha S h=\phi^{\prime}(1)$ & $\alpha$ & $\alpha N u=\beta^{\prime}(1)$ & $\alpha S h=\phi^{\prime}(1)$ \\
\hline $0^{\circ}$ & 0.670000 & -0.133889 & $0^{\circ}$ & 0.670000 & -0.133889 \\
$-2^{\circ}$ & 0.698657 & -0.120838 & $2^{\circ}$ & 0.645605 & -0.146431 \\
$-4^{\circ}$ & 0.728034 & -0.107761 & $4^{\circ}$ & 0.628332 & -0.158178 \\
$-6^{\circ}$ & 0.754221 & -0.095311 & $6^{\circ}$ & 0.620146 & -0.168901 \\
\hline
\end{tabular}

Table 7. Variation in Nusselt number and Sherwood number with varying $H a$ when $R e=25, E_{c}=1, S_{c}=S_{r}=0.2, D_{f}=P_{r}=0.5, \gamma=0.1$.

\begin{tabular}{ccccc}
\hline \multicolumn{4}{c}{$\alpha=-5^{\circ}$} & $\alpha=5^{\circ}$ \\
\hline$H a$ & $\alpha N u=\beta^{\prime}(1)$ & $\alpha S h=\phi^{\prime}(1)$ & $\alpha N u=\beta^{\prime}(1)$ & $\alpha S h=\phi^{\prime}(1)$ \\
0 & 0.733702 & -0.106982 & 0.601853 & -0.171361 \\
200 & 0.747908 & -0.096138 & 0.642952 & -0.156091 \\
400 & 0.754835 & -0.086380 & 0.678122 & -0.141432 \\
600 & 0.755444 & -0.077668 & 0.706215 & -0.127721 \\
\hline
\end{tabular}

Table 8. Variation in Nusselt number and Sherwood number with varying $S_{c}$ when $R e=25, E_{c}=1, S_{r}=0.2, D_{f}=P_{r}=0.5, \gamma=0.1$.

\begin{tabular}{ccccc}
\hline \multicolumn{3}{c}{$\alpha=-5^{\circ}$} & \multicolumn{3}{c}{$\alpha=5^{\circ}$} \\
\hline$S_{c}$ & $\alpha N u=\beta^{\prime}(1)$ & $\alpha S h=\phi^{\prime}(1)$ & $\alpha N u=\beta^{\prime}(1)$ & $\alpha S h=\phi^{\prime}(1)$ \\
0.5 & 0.744752 & -0.101423 & 0.630359 & -0.163681 \\
1.0 & 0.749722 & -0.203428 & 0.642586 & -0.329127 \\
1.5 & 0.754690 & -0.306011 & 0.654809 & -0.496338 \\
2.0 & 0.759656 & -0.409171 & 0.667028 & -0.665313 \\
\hline
\end{tabular}

Table 9. Variation in Nusselt number and Sherwood number with varying $S_{r}$ when $R e=25, E_{c}=1, S_{c}=0.2, D_{f}=P_{r}=0.5, \gamma=0.1$.

\begin{tabular}{ccccc}
\hline \multicolumn{5}{c}{$\alpha=-5^{\circ}$} \\
$S_{r}$ & $\alpha N u=\beta^{\prime}(1)$ & $\alpha S h=\phi^{\prime}(1)$ & $\alpha N u=\beta^{\prime}(1)$ & $\alpha S h=\phi^{\prime}(1)$ \\
0.5 & 0.744695 & -0.050600 & 0.630303 & -0.081579 \\
1.0 & 0.749573 & -0.101190 & 0.642440 & -0.163447 \\
1.5 & 0.754450 & -0.151922 & 0.654576 & -0.245755 \\
2.0 & 0.759327 & -0.202797 & 0.666713 & -0.328503 \\
\hline
\end{tabular}

Table 10. Variation in Nusselt number and Sherwood number with varying $P_{r}$ when $R e=25, E_{c}=1, S_{r}=S_{c}=0.2, D_{f}=0.5, \gamma=0.1$.

\begin{tabular}{|c|c|c|c|c|}
\hline & $\alpha=-5^{\circ}$ & & $\alpha=5^{\circ}$ & \\
\hline$P_{r}$ & $\alpha N u=\beta^{\prime}(1)$ & $\alpha S h=\phi^{\prime}(1)$ & $\alpha N u=\beta^{\prime}(1)$ & $\alpha S h=\phi^{\prime}(1)$ \\
\hline 0.5 & 0.741769 & -0.020314 & 0.623021 & -0.032670 \\
\hline 1.0 & 1.487440 & -0.040499 & 1.255751 & -0.065259 \\
\hline 1.5 & 2.237014 & -0.060706 & 1.898192 & -0.097917 \\
\hline 2.0 & 2.990488 & -0.080937 & 2.550341 & -0.130647 \\
\hline
\end{tabular}


Table 11. Variation in Nusselt number and Sherwood number with varying $E_{c}$ when $R e=25, S_{r}=S_{c}=0.2, D_{f}=P_{r}=0.5, \gamma=0.1$.

\begin{tabular}{|c|c|c|c|c|}
\hline & $\alpha=-5^{\circ}$ & & $\alpha=5^{\circ}$ & \\
\hline$E_{c}$ & $\alpha N u=\beta^{\prime}(1)$ & $\alpha S h=\phi^{\prime}(1)$ & $\alpha N u=\beta^{\prime}(1)$ & $\alpha S h=\phi^{\prime}(1)$ \\
\hline 0.5 & 0.370903 & -0.010234 & 1.059363 & -0.016412 \\
\hline 1.0 & 0.741769 & -0.020314 & 2.118688 & -0.032670 \\
\hline 1.5 & 1.112635 & -0.030395 & 3.178012 & -0.048929 \\
\hline 2.0 & 1.483501 & -0.040476 & 4.237338 & -0.065188 \\
\hline
\end{tabular}

Table 12. Variation in Nusselt number and Sherwood number with varying $D_{f}$ when $R e=25, E_{c}=1, S_{r}=S_{c}=0.2, P_{r}=0.5, \gamma=0.1$.

\begin{tabular}{ccccc}
\hline \multicolumn{4}{c}{$\alpha=-5^{\circ}$} & \multicolumn{3}{c}{$\alpha=5^{\circ}$} \\
\hline$D_{f}$ & $\alpha N u=\beta^{\prime}(1)$ & $\alpha S h=\phi^{\prime}(1)$ & $\alpha N u=\beta^{\prime}(1)$ & $\alpha S h=\phi^{\prime}(1)$ \\
0.5 & 0.741769 & -0.020314 & 0.623021 & -0.032670 \\
1.0 & 0.743759 & -0.020339 & 0.627914 & -0.032706 \\
1.5 & 0.745747 & -0.020338 & 0.632806 & -0.032742 \\
2.0 & 0.747736 & -0.020350 & 0.637691 & -0.032778 \\
\hline
\end{tabular}

Table 13. Variation in Nusselt number and Sherwood number with varying $\gamma$ when $R e=25, E_{c}=1, S_{r}=S_{c}=0.2, D_{f}=P_{r}=0.5$.

\begin{tabular}{ccccc}
\hline \multicolumn{4}{c}{$\alpha=-5^{\circ}$} & $\alpha=5^{\circ}$ \\
\hline$\gamma$ & $\alpha N u=\beta^{\prime}(1)$ & $\alpha S h=\phi^{\prime}(1)$ & $\alpha N u=\beta^{\prime}(1)$ & $\alpha S h=\phi^{\prime}(1)$ \\
0.2 & 0.741807 & -0.020467 & 0.623059 & -0.032821 \\
0.4 & 0.741883 & -0.020771 & 0.623134 & -0.033125 \\
0.6 & 0.741959 & -0.021075 & 0.623201 & -0.033429 \\
0.8 & 0.742035 & -0.213797 & 0.623285 & -0.033732 \\
\hline
\end{tabular}

In addition to this section highlights the major outcomes of the analytical study presented by new algorithm. The analysis of the variations in temperature and concentration profiles for different parameters is prepared. For that purpose, Figures are plotted for varying several parameters. Moreover we have been divided this section into two subsections follow as.

- Channel divergent $(\alpha>0)$.

In Figures 2-9 are plotted to show the behavior curves of velocity, temperature and concentration profiles under the impact of different physical parameters. An increasing the opening angle $\alpha$ gives variations in velocity, temperature and concentration profiles as displayed in Figure 2. The influence of parameter $\alpha$ on the velocity field $f(\eta)$ for divergent channel causes more effect at the middle channel as well as it represents as a maximum position at the central line (when $\eta=0$ ). It also has least effect in part near the walls (when 
$\eta=-1,1)$. High temperature in the central region of the channel is conspicuously clear and the maximum temperature lies there too. While the results of angle opening $\alpha$ on concentration profile show that the increase in $\alpha$ gives a decreased concentration profile. The central portion of the channel is more effect, while the portion of the near of walls the concentration is less affected. Effects of the increase Reynolds number $R e$ in $f(\eta), \beta(\eta)$ and $\phi(\eta)$ are observed in Figure 3. The velocity field $f(\eta)$ is decreasing with increasing Reynold number and clearly shows that the highest level reaches the central part (when $\eta=0$ ). A rise in temperature profile $\beta(\eta)$ leads to an increase in $R e$ as for the concentration profile $\phi(\eta)$, it is in a state of decrease when there is an increase in $R e$. The prominent appearance in the central part can be observed in the lowest effect. The behavior of temperature distribution under the influence of Hartmann number $\mathrm{Ha}$ can be seen from Figure 4. The velocity distribution remains unchanged with the increase Hartmann. This figure gives a clear picture of how a stronger magnetic field can lead to a change in the temperature of the fluid. But Hartmann number gives a simple rise to the concentration $\phi(\eta)$ although this increase is simple, however can be a way to control the concentration of the fluid. In Figure 5 the change in temperature and concentration with an increase in Soret number $S r$ are plotted. A rise in temperature and a drop in concentration at the central portion of the channel are observed. In fact that stronger viscous forces are responsible for these phenomena. Impact of the Schmidt number $S c$ on temperature and concentration are demonstrated in Figure 6, with note that the effect $S r$ is similar to the effect of the Soret number $S r$. Figure 7 is plotted the effect of growing values of Prandtl number on temperature profile. Arise in temperature of the fluid with increasing $\operatorname{Pr}$ can be seen. Figure 8 explains that change in temperature with rising Eckert number $E c$. Again the increase in temperature was observed to increase $E c$. The effect of Eckert number on $\phi(\eta)$ for diverging channel, an increase in $E c$ decreases the concentration profile. This shows, the strong viscous forces are responsible for a rise temperature of the fluid and this rise is a lot of effect at the central portion. In Figure 9 the changes of chemical reaction $\gamma$ and Dufour number $D_{f}$ on $\beta$ and $\phi(\eta)$ are presented. Dufour number arises due to the concentration gradient present in energy equation. The main variations in temperature are in central portion of the channel, where the variation in temperature near the walls is almost negligible, which can be said, the stronger concentration results in higher temperature values. Also this figure demonstrates that the increasing of $\gamma$ lead to decrease the concentration profile.

- Channel convergent $(\alpha<0)$.

For the converging channel, the variations in velocity, temperature and concentration profile due to the varying parameters are depicted in Figures 10-17, the behavior of velocity and temperature for changing angle opening $\alpha$ and Reynolds number $R e$ is quite opposite to the behavior of $f(\eta), \beta(\eta)$ and $\phi(\eta)$ in diverging channel as seen in Figure 10 and Figure 11. On that other hand, Figures 11-17 tell the effect of Harmann, Eckert. 

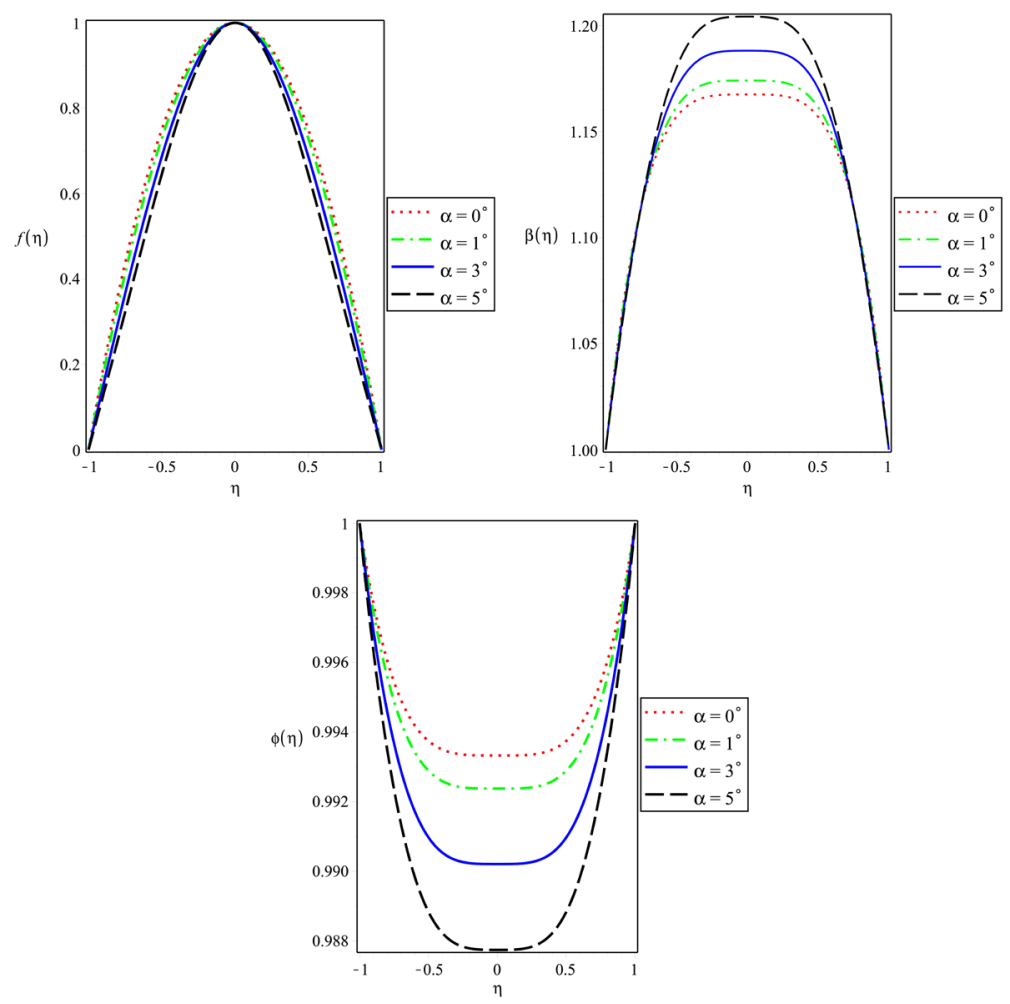

Figure 2. $f(\eta), \beta(\eta), \phi(\eta)$ for the value $R e=50, H a=100, \operatorname{Pr}=D_{f}=0.5$, $E c=1, S c=S r=0.2, \gamma=0.1$, when the angle $\alpha$ is varied.
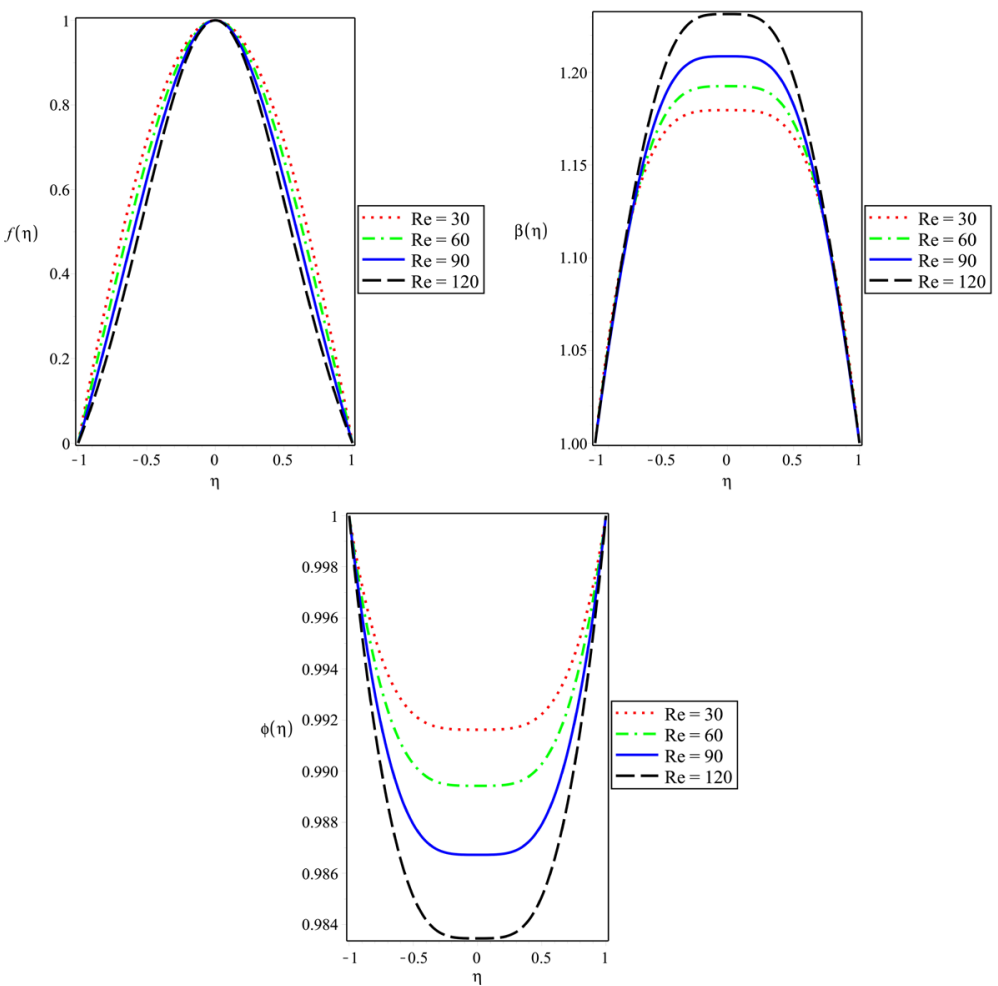

Figure 3. $f(\eta), \beta(\eta), \phi(\eta)$ for the value $H a=100, \operatorname{Pr}=D_{f}=0.5, \quad E c=1$, $S c=S r=0.2, \quad \gamma=0.1, \quad \alpha=3^{\circ}$ when the Reynolds number $R e$ is varied. 

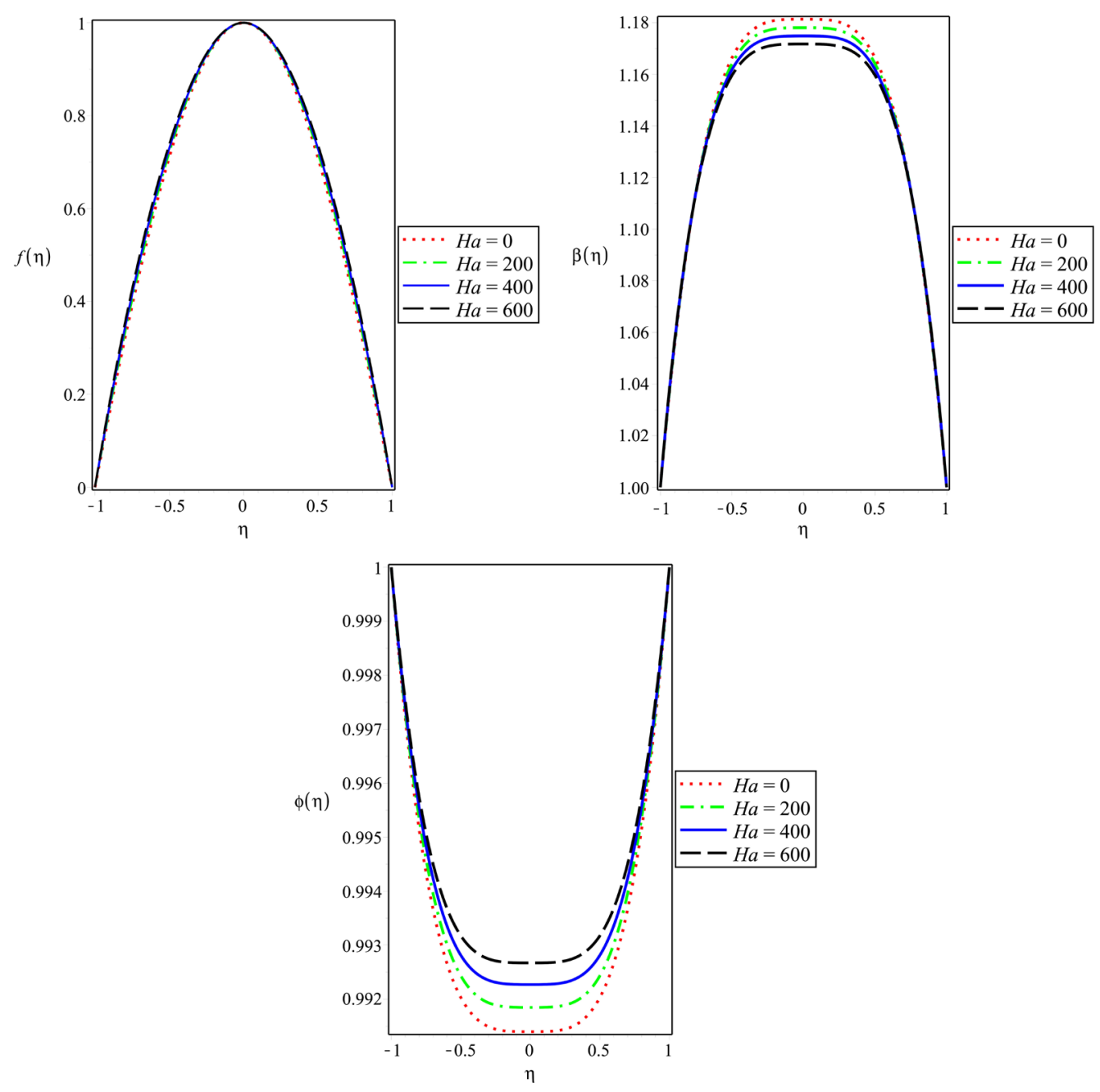

Figure 4. $f(\eta), \beta(\eta), \phi(\eta)$ for the value $R e=50, \operatorname{Pr}=D_{f}=0.5, E c=1, \quad S c=S r=0.2$, $\gamma=0.1, \alpha=3^{\circ}$ when the Harmann number $H a$ is varied.
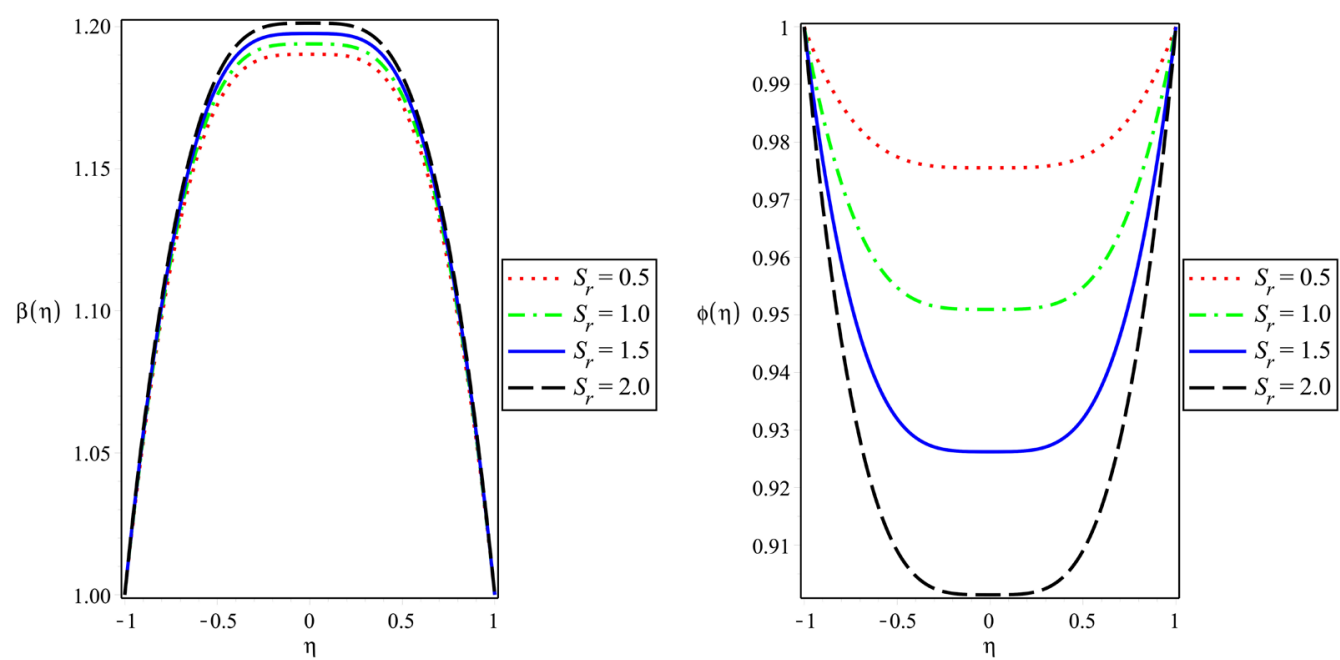

Figure 5. $f(\eta), \beta(\eta), \phi(\eta)$ for the value $\operatorname{Re}=50, H a=100, \quad \operatorname{Pr}=D_{f}=0.5, \quad E c=1$, $S c=0.2, \gamma=0.1, \alpha=3^{\circ}$ when the Soret number $S r$ is varied. 

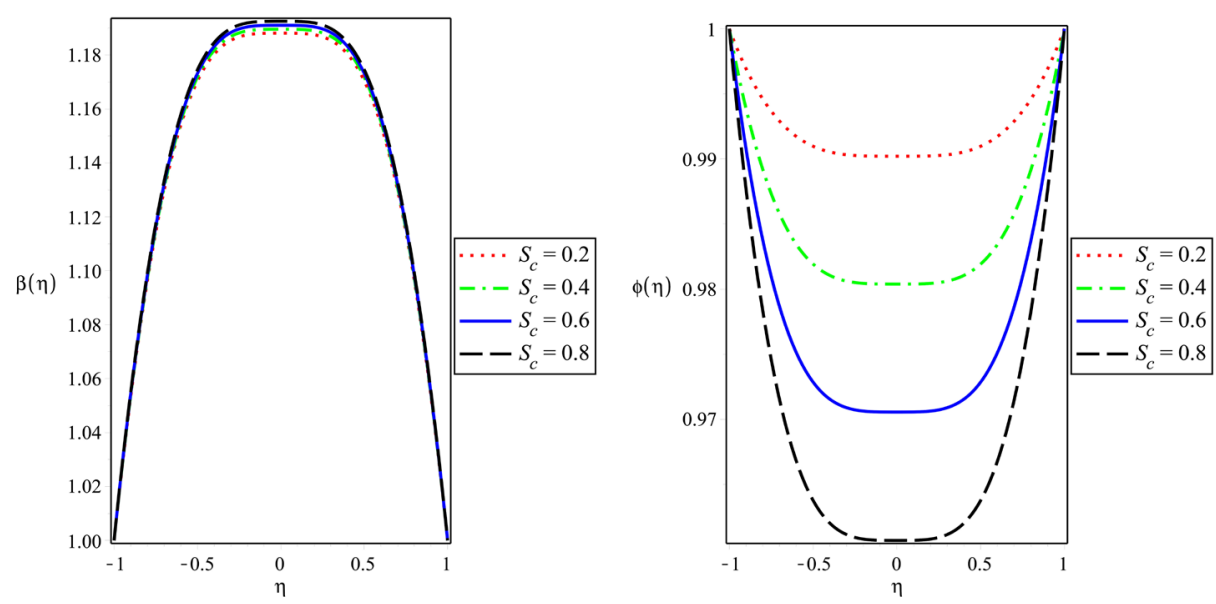

Figure 6. $f(\eta), \beta(\eta), \phi(\eta)$ for the value $R e=50, H a=100, \operatorname{Pr}=D_{f}=0.5, E c=1$, $S r=0.2, \gamma=0.1, \quad \alpha=3^{\circ}$ when the Schmidt number $S c$ is varied.
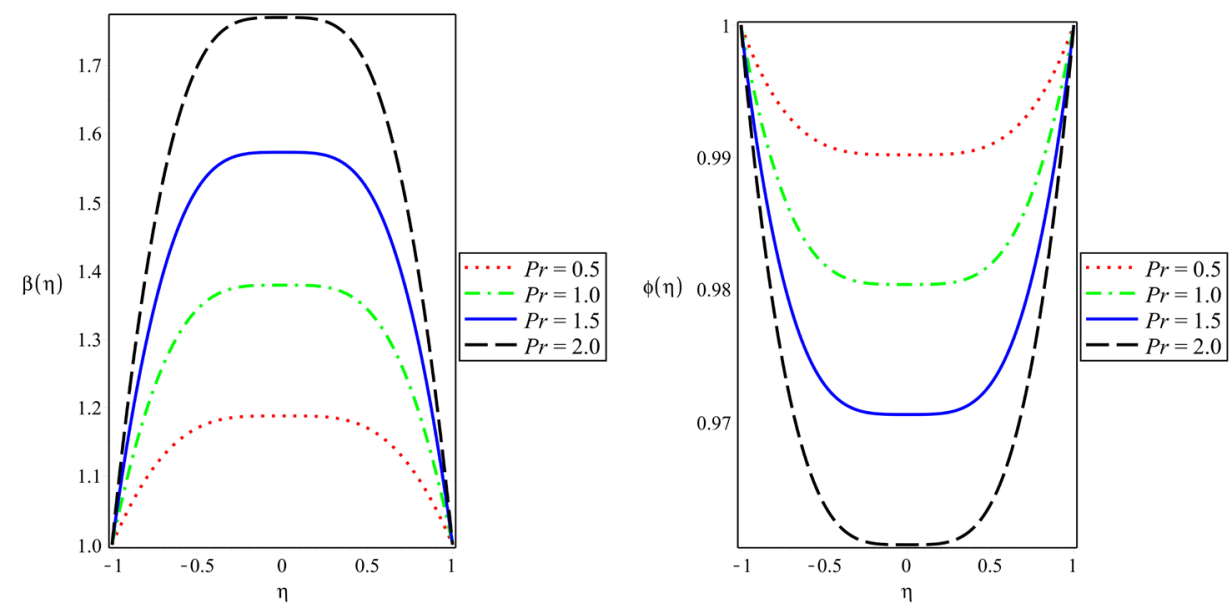

Figure 7. $f(\eta), \beta(\eta), \phi(\eta)$ for the value $R e=50, H a=100, D_{f}=0.5, E c=1$, $S c=S r=0.2, \quad \gamma=0.1, \quad \alpha=3^{\circ}$ when the Prandtl number $\operatorname{Pr}$ is varied.
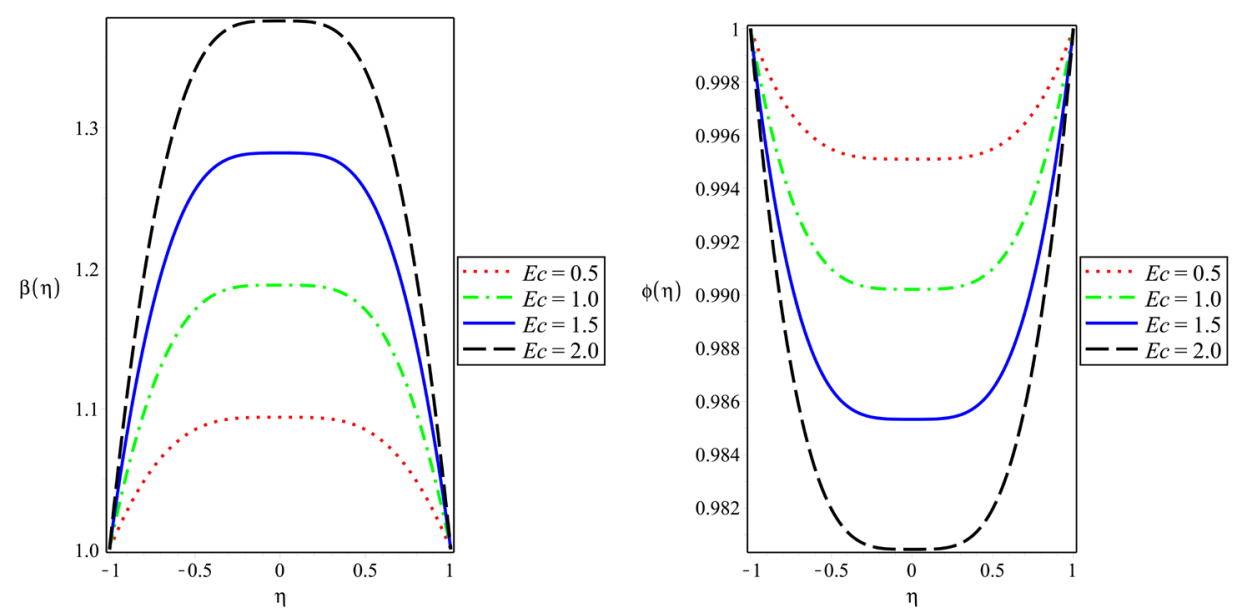

Figure 8. $\beta(\eta), \phi(\eta)$ for the value $R e=50, H a=100, \operatorname{Pr}=D_{f}=0.5, S c=S r=0.2$, $\gamma=0.1, \alpha=3^{\circ}$ when the Eckert number $E c$ is varied. 

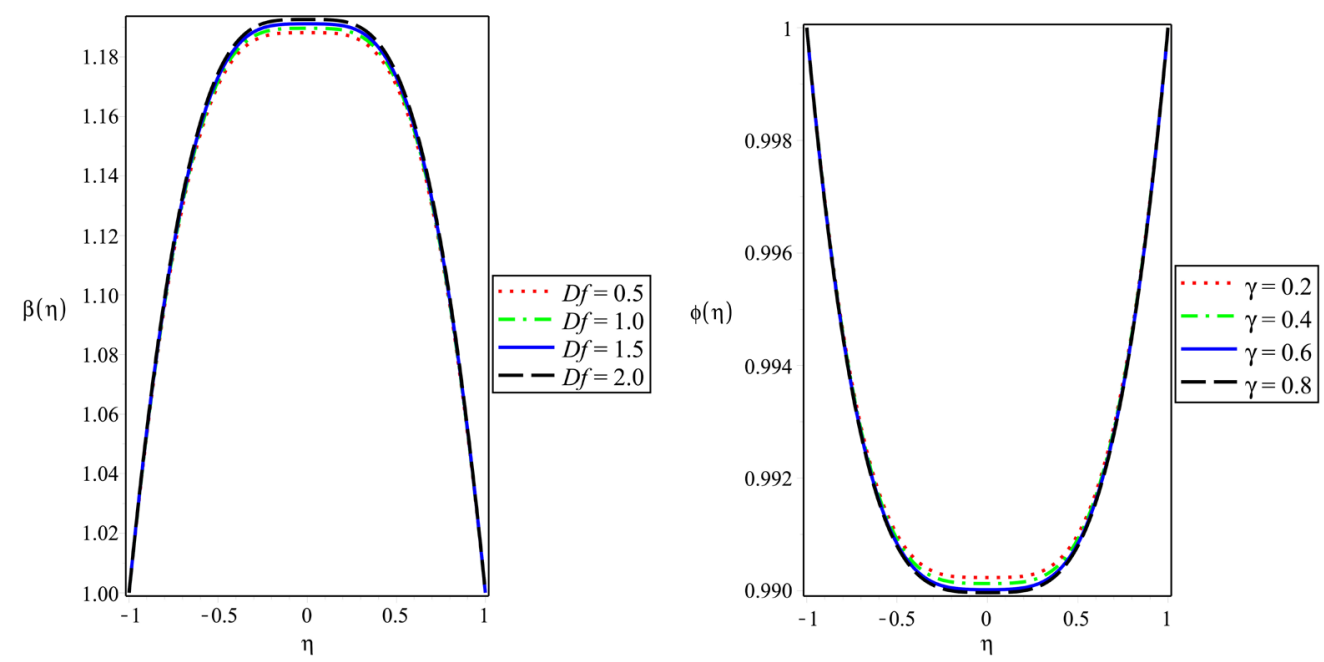

Figure 9. $\beta(\eta), \phi(\eta)$ for the value $R e=50, H a=100, P r=0.5, E c=1, S c=S r=0.2$, $\gamma=0.1, \alpha=3^{\circ}$ when the Dufour number $D_{f}$ and chemical reaction parameter $\gamma$ are varied.
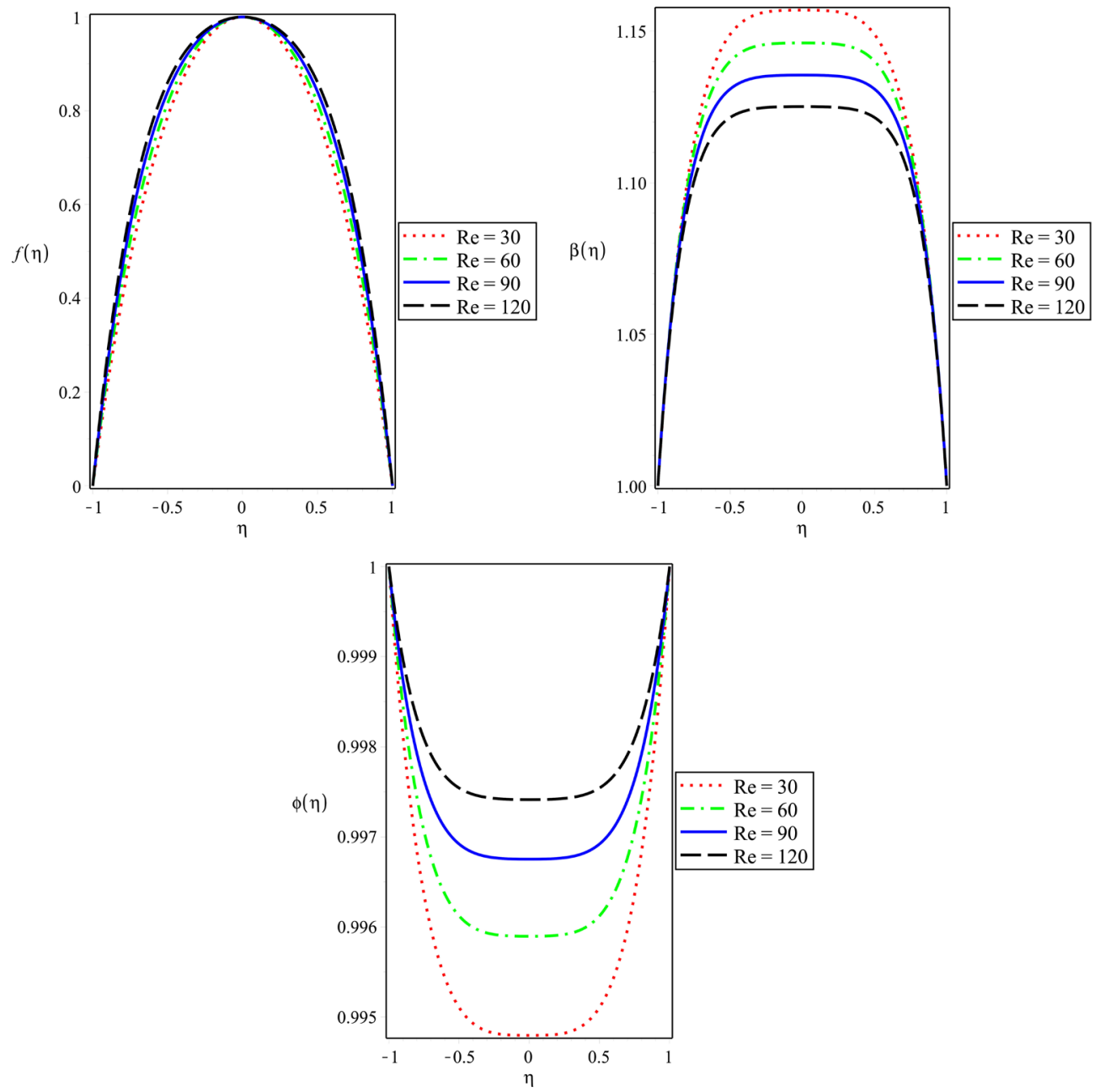

Figure 10. $f(\eta), \beta(\eta), \phi(\eta)$ for the value $H a=100, P r=D_{f}=0.5, E c=1, S c=S r=0.2$, $\gamma=0.1, \alpha=-3^{\circ}$ when the Reynolds number $\operatorname{Re}$ is varied. 

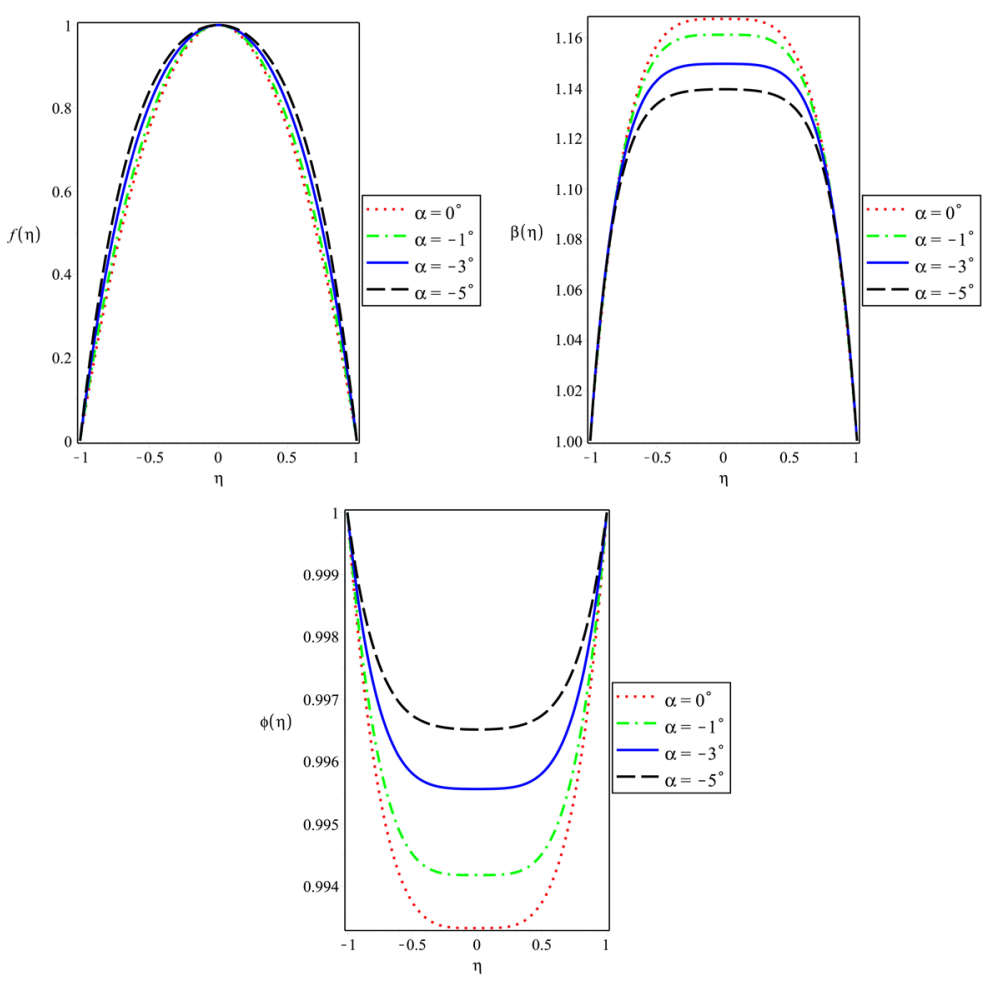

Figure 11. $f(\eta), \beta(\eta), \phi(\eta)$ for the value $R e=50, H a=100, \operatorname{Pr}=D_{f}=0.5$, $E c=1, S c=S r=0.2, \gamma=0.1$, when the angle $\alpha$ is varied.
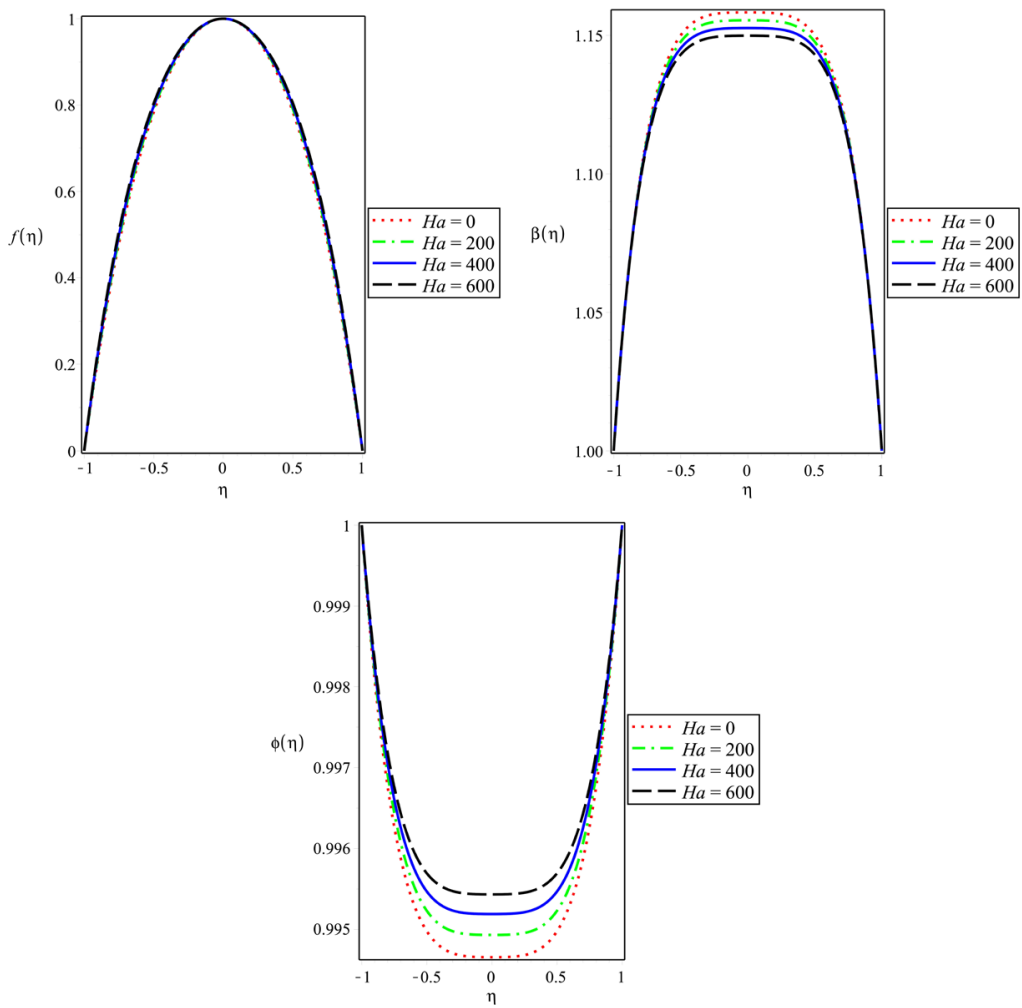

Figure 12. $f(\eta), \beta(\eta), \phi(\eta)$ for the value $\operatorname{Re}=30, \operatorname{Pr}=D_{f}=0.5, E c=1$, $S c=S r=0.2, \gamma=0.1, \alpha=-3^{\circ}$ when the Harmann number $H a$ is varied. 

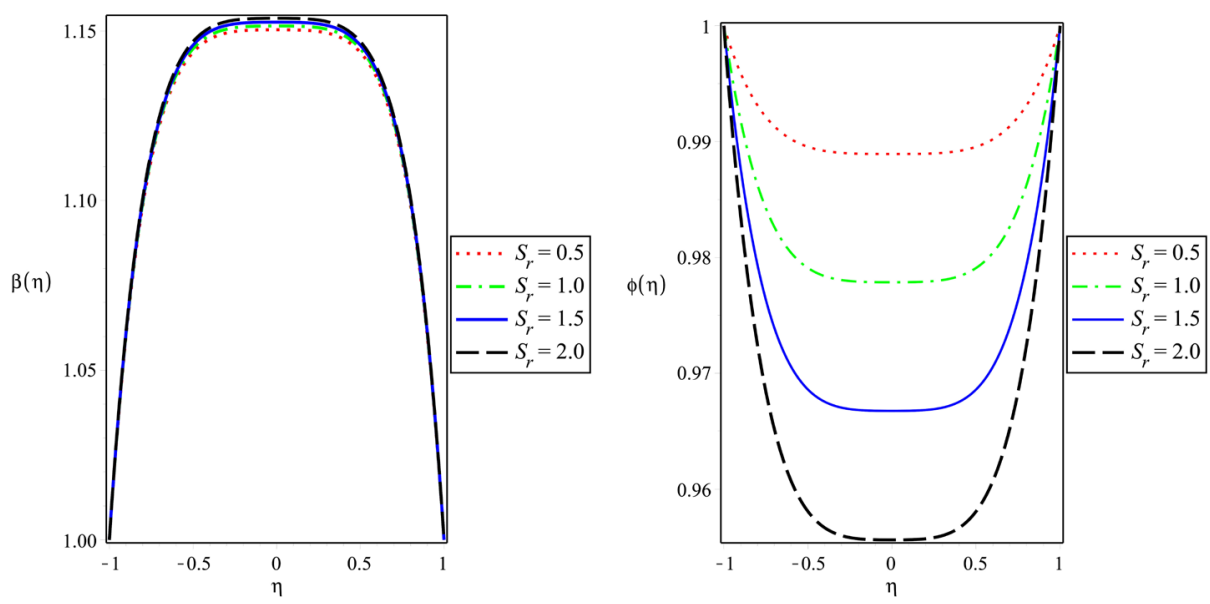

Figure 13. $f(\eta), \beta(\eta), \phi(\eta)$ for the value $R e=50, H a=100, \quad \operatorname{Pr}=D_{f}=0.5$, $E c=1, S c=0.2, \gamma=0.1, \quad \alpha=-3^{\circ}$ when the Soret number $S r$ is varied.
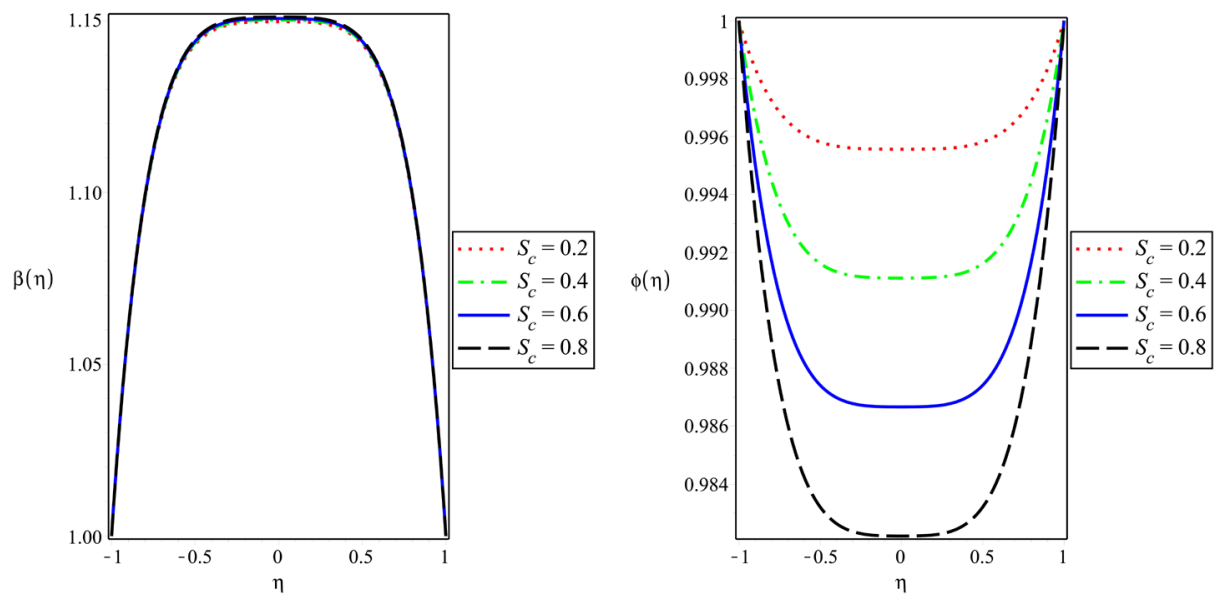

Figure 14. $f(\eta), \beta(\eta), \phi(\eta)$ for the value $\operatorname{Re}=50, H a=100, \operatorname{Pr}=D_{f}=0.5$, $E c=1, S r=0.2, \gamma=0.1, \alpha=-3^{\circ}$ when the schmidt number $S c$ is varied.
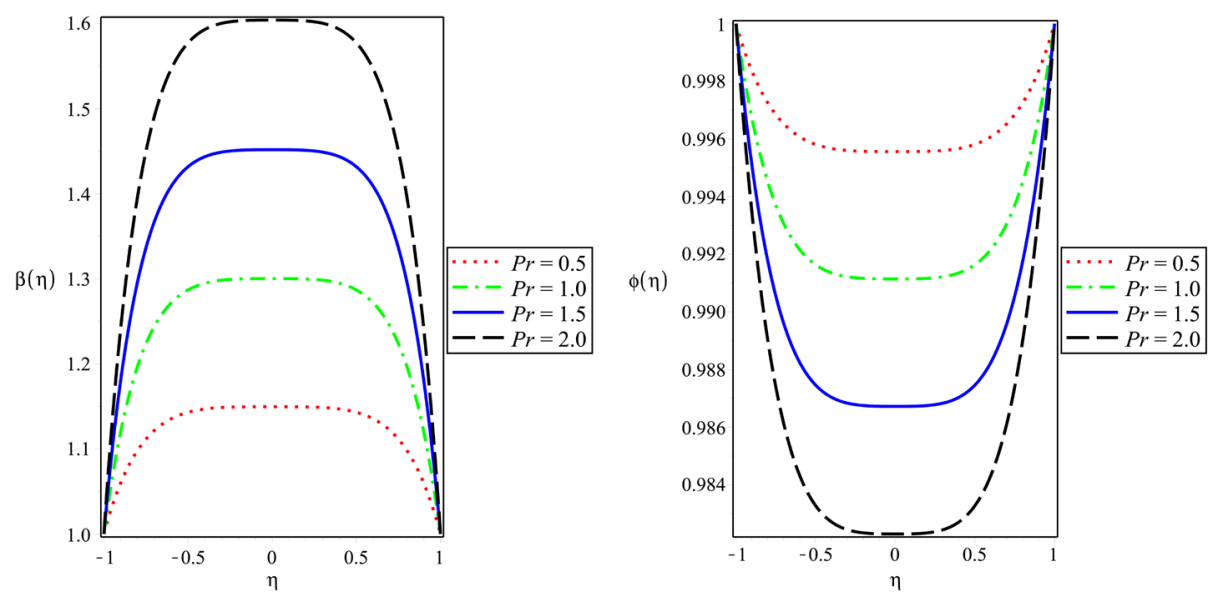

Figure 15. $f(\eta), \beta(\eta), \phi(\eta)$ for the value $R e=50, H a=100, D_{f}=0.5, E c=1$, $S c=S r=0.2, \gamma=0.1, \quad \alpha=-3^{\circ}$ when the Prandtl number $\operatorname{Pr}$ is varied. 

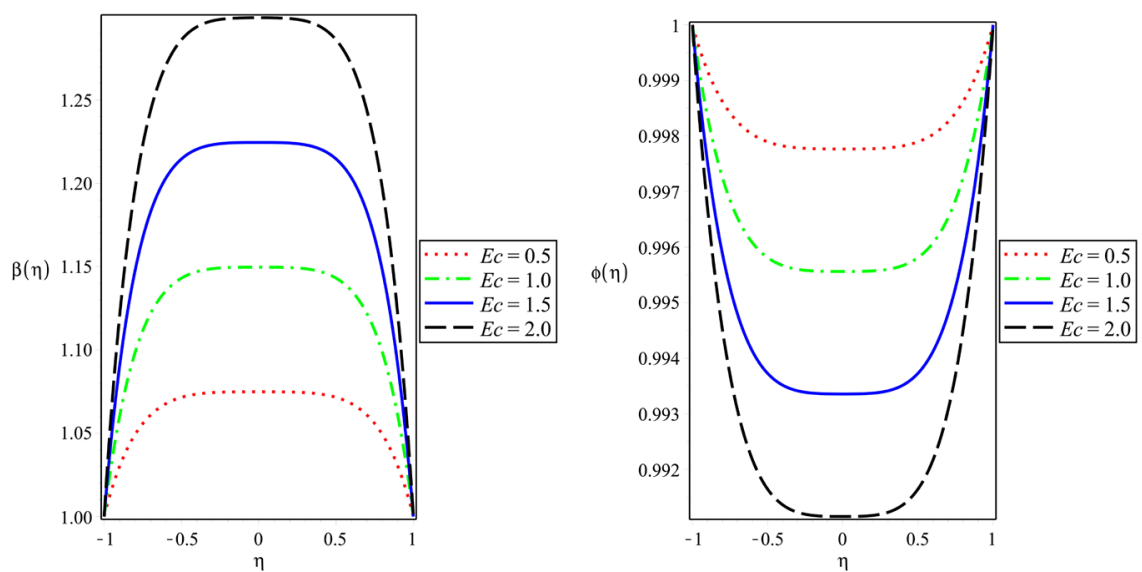

Figure 16. $f(\eta), \beta(\eta), \phi(\eta)$ for the value $R e=50, H a=100, \operatorname{Pr}=D_{f}=0.5$, $S c=S r=0.2, \gamma=0.1, \quad \alpha=-3^{\circ}$ when the Eckert number $E c$ is varied.
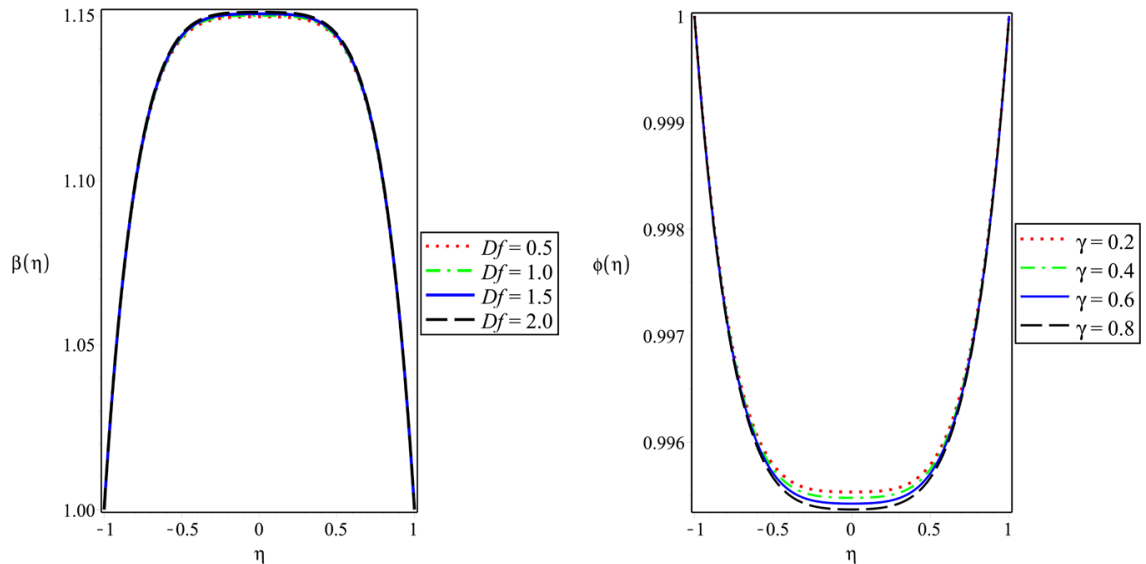

Figure 17. $f(\eta), \beta(\eta), \phi(\eta)$ for the value $R e=50, H a=100, \operatorname{Pr}=0.5, E c=1$, $S c=S r=0.2, \gamma=0.1, \alpha=-3^{\circ}$ when the Dufour number $D_{f}$ and chemical reaction parameter $\gamma$ are varied.

Prandtl and Dufour numbers on the temperature profile is similar for the effect in diverging channel. Also these Figures demonstrated the concentration profile possess same effect when there are changing in Hartmann number, Schimdt number, Soret number and chemical reaction parameter. Physical explanations can be provided the temperature profile show that the temperature at the central region increases with increasing angle opening. This can be attributed to that for fixed Reynold number, increasing angle opening leads to increase the cross-sectional flow area. This in turn leads to decrease the flow velocity and this mean the flow will be decelerated. Therefore, the heat dissipation will be reduced which leads to increase the temperature of the fluid. The inertia force of the fluid increases with increasing Reynold number which leads to enhance the parabolic behavior (increasing central temperature) for diverging channel with the opposite view in converging channel. Hartmman number increase in this case Lorentz force is also increasing for diverging and converging 
channels. This force imports extra drag to the flow. Therefore the temperature profile becomes more flat which means decreasing the temperature within the central region. The thin boundary layers that are near to the wall lead to that the temperature gradient at the highest level. Furthermore to the existence of the thick boundary layer in central region lead to that the temperature gradient at low level. Eckert number increases with increased temperature and thus produces an increase in Kinetic energy. The change of the temperature profile with Prandtl number, and the increase of temperature with Prandtl number result from increasing of the momentum diffusivity. The Dufour number shows to increase shows less effect on temperature, increasing Dufour leads to increase the thermal energy of the fluid thus the temperature increase. The rate of most chemical reactions increases with a decrease the concentration of reactants. As for temperature, it increases if a reaction is heat-emitting and decreases when the reaction absorbs heat.

\section{Conclusions}

In this paper, the unsteady and two-dimensional magneto hydrodynamic (MHD) flow of viscous fluid in a channel with non-parallel plates is studied analytically using a new algorithm. The solution obtained by new algorithm is an infinite power series for appropriate initial approximation. The construction of this algorithm possessed good convergent series and the convergence of the results is explicitly shown. Graphical results and tables are presented to investigate the influence of physical parameters on velocity, temperature and concentration. Analysis of the converge confirms that the new algorithm is an efficient technique as compared to Range-Kutta algorithm with help of Shooting algorithm. The new algorithm that is widely applied to solve ordinary differential equations lead to the solutions resulting from this algorithm is compatible with numerical solution. Effects of different parameters on temperature and concentration profiles are analyzed and presented graphically. The conclusions can be drawn from the analysis presented:

- The behavior of temperature and concentration profiles are the same results $\alpha, \operatorname{Re}, E_{c}, P_{r}$ and $D_{f}$ for diverging channel.

- Hartmann number $\mathrm{Ha}$ can be used to reduce the temperature of the flow fluid. Also, concentration of the fluid can also be controlled by employing a strong magnetic field.

- For converging channel, the variations in temperature are opposite for diverging channel with an increase in channel opening $\alpha$ and $R e$.

- For diverging channel, Nusselt number drops with a rise in angle opening and increases with a rise in Reynolds number and behaves oppositely for convergent channel.

- Increase in heat transfer rate is observed for increasing $P_{r}, E_{c}, S_{r}, S_{c}$, $D_{f}$ and $\gamma$ in both channels.

- Increase in Reynolds number and Angle opening gives a drop to mass trans- 
fer rate for diverging channel and a rise for the converging channel.

- The rate of mass transfer decreased for both channels with an increase in Schmidt, Soret, Prandtl, Eckert, Dufour numbers and chemical reaction parameter.

- Results obtained by new algorithm are in excellent agreement with numerical solution obtained.

\section{Conflicts of Interest}

The authors declare no conflicts of interest regarding the publication of this paper.

\section{References}

[1] Eckert, E.R.G. and Drake, R.M. (1972) Analysis of Heat and Mass Transfer. McGraw-Hill Book Company, New York.

[2] Olajuwon, B.I. (2011) Convection Heat and Mass Transfer in a Hydromangetic Flow of a Second Grade Fluid in the Presence of Thermal Radiation and Thermal Diffusion. International Communication in Heat and Mass Transfer, 38, 377-382. https://doi.org/10.1016/j.icheatmasstransfer.2010.11.006

[3] Kumar, A.G.V., Goud, Y.R. and Varma, S.V.K. (2012) Thermal Diffusion and Radiation Effects on Unsteady MHD Flow through Porous Medium with Variable Temperature and Mass Diffusion in the Presence of Heat Source Sink. Advance in Applied Science Research, 3, 1494-1506.

[4] Davidson, P.A. (2006) An Introduction to Magnetohydrodynamics. Cambridge University Press, Cambridge.

[5] Gailitis, A. and Lielausis, O. (2006) On a Possibility to Reduce the Hydrodynamical Resistance of a Plate in an Electrolyte. Applied Magnetohydrodynamics, 12, 143-146.

[6] Henoch, C. and Stace, J. (1995) Experimental Investigation of a Salt-Water Turbulent Boundary-Layer Modified by an Applied Stream Wise Magneto Hydrodynamic Body Force. Physics of Fluids, 7, 1371-1383. https://doi.org/10.1063/1.868525

[7] Ellahi, R., Raza, M. and Vafai, K. (2012) Series Solutions of Non-Newtonian Nanofluids with Reynolds' Model and Vogel's Model by Means of the Homotopy Analysis Method. Mathematical and. Computer Modelling, 55, 1876-1891. https://doi.org/10.1016/j.mcm.2011.11.043

[8] Khan, U., Ahmed, N. and Tauseef Mohyud-Din, S. (2016) Thermo-Diffusion, Diffusion-Thermo and Chemical Reaction Effects on MHD Flow of Viscous Fluid in Divergent and Convergent Channels. Chemical Engineering Science, 141, 17-27. https://doi.org/10.1016/j.ces.2015.10.032

[9] Hatami, M., Sheikholeslami, M., Hosseini, M. and Ganji, D.D. (2014) Analytical Investigation of MHD Nanofluid Flow in Non-Parallel Walls. Journal of Molecular Liquids, 194, 251-259. https://doi.org/10.1016/j.molliq.2014.03.002

[10] Hatami, M., Sheikholeslami, M. and Ganji, D.D. (2014) Nanofluid Flow and Heat Transfer in an Asymmetric Porous Channel with Expanding or Contracting Wall. Journal of Molecular Liquids, 195, 230-239. https://doi.org/10.1016/j.molliq.2014.02.024https://doi.org/10.1016/j.molliq.2016.10 .073

[11] Turkyilmazoglu, M. (2014) Extending the Traditional Jeffery-Hamel Flow to Stret- 
chable Convergent/Divergent Channels. Computers and Fluids, 100, 196-203.

https://doi.org/10.1016/j.compfluid.2014.05.016

[12] Asadullaha, A.M., Khan, U., Ahmed, N. and Tauseef Mohyud-Dinb, S. (2016) Analytical and Numerical Investigation of Thermal Radiation Effects on Flow of Viscous Incompressible Fluid with Stretchable Convergent/Divergent Channels. Journal of Molecular Liquids, 224, 768-775. https://doi.org/10.1016/j.molliq.2016.10.073

[13] Asadullah, M., Khan, U., Manzoor, R., Ahmed, N. and Mohyud-din, S.T. (2013) MHD Flow of a Jeffery Fluid in Converging and Diverging Channels. International Journal of Modern Mathematical Sciences, 6, 92-106.

[14] Hayat, T., Nawaz, M. and Sajid, M. (2012) Effect of Heat Transfer on the Flow of a Second-Grade Fluid in Divergent/Convergent Channel. International Journal for Numerical Methods in Fluids, 64, 761-776. 